
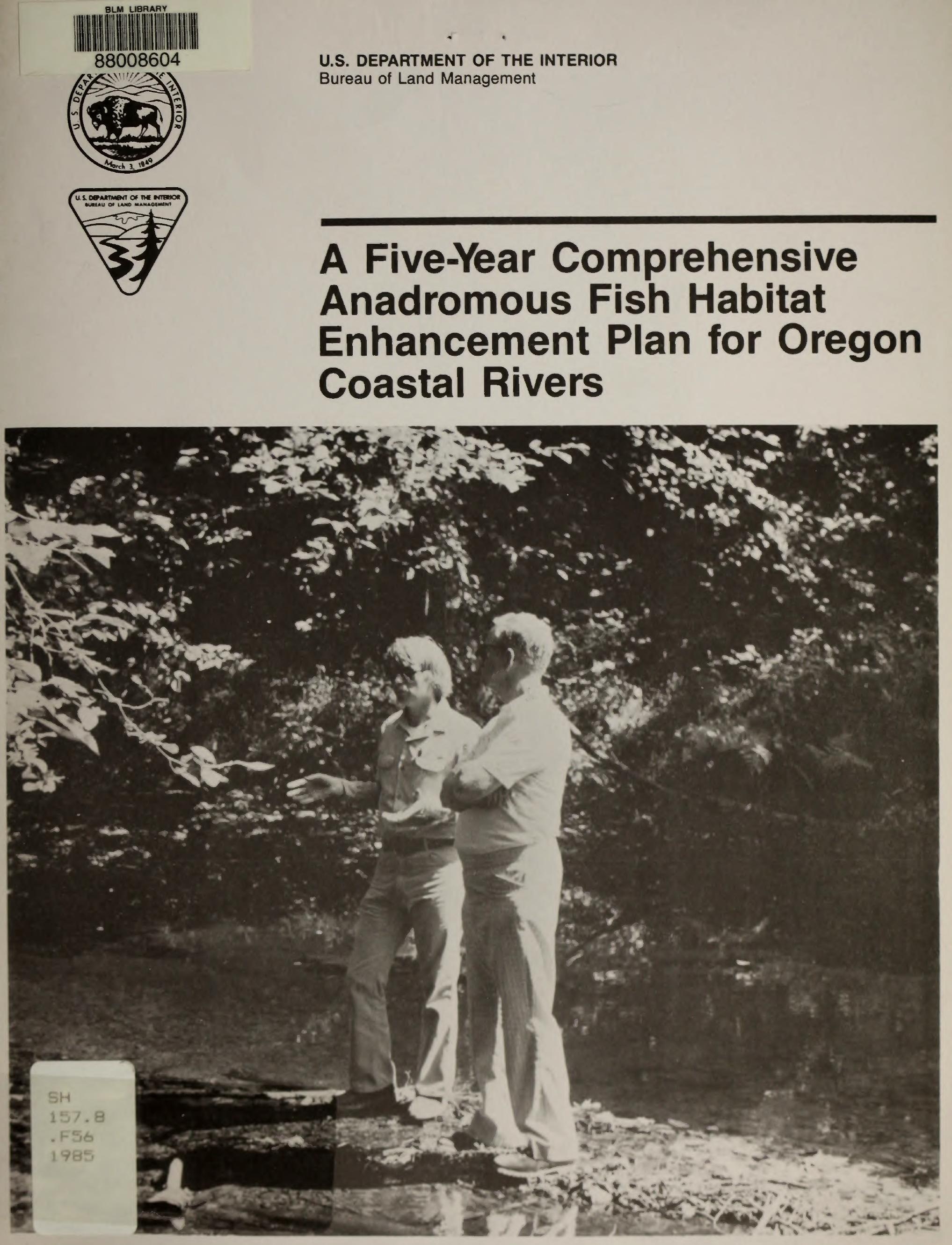


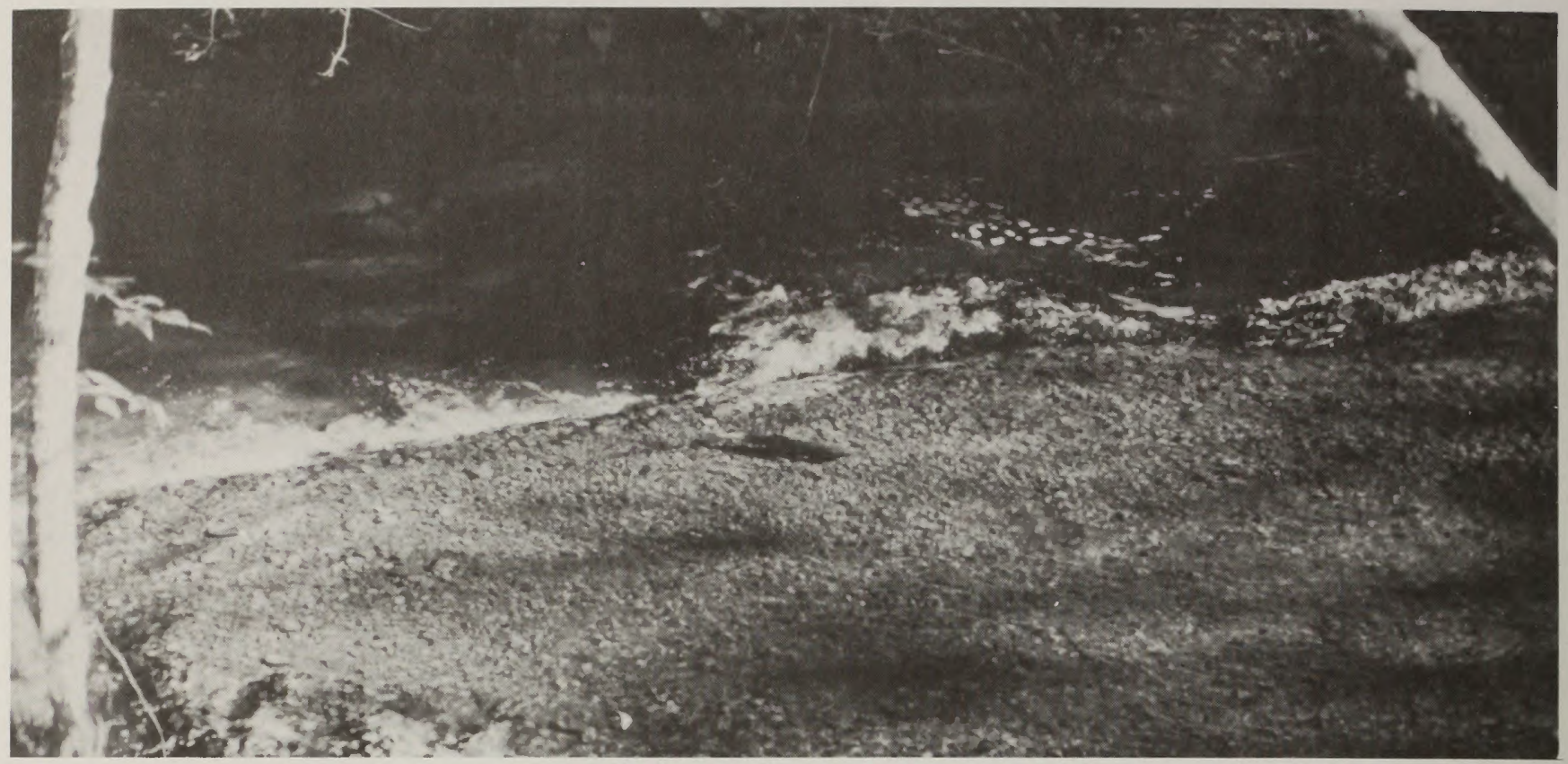

A large spawned-out chinook salmon near one of several completed redds (nests) located just upstream from a gabion structure. Note excellent spawning gravel and large pool below the gabion used both by young fish for rearing and adult fish for resting and hiding.

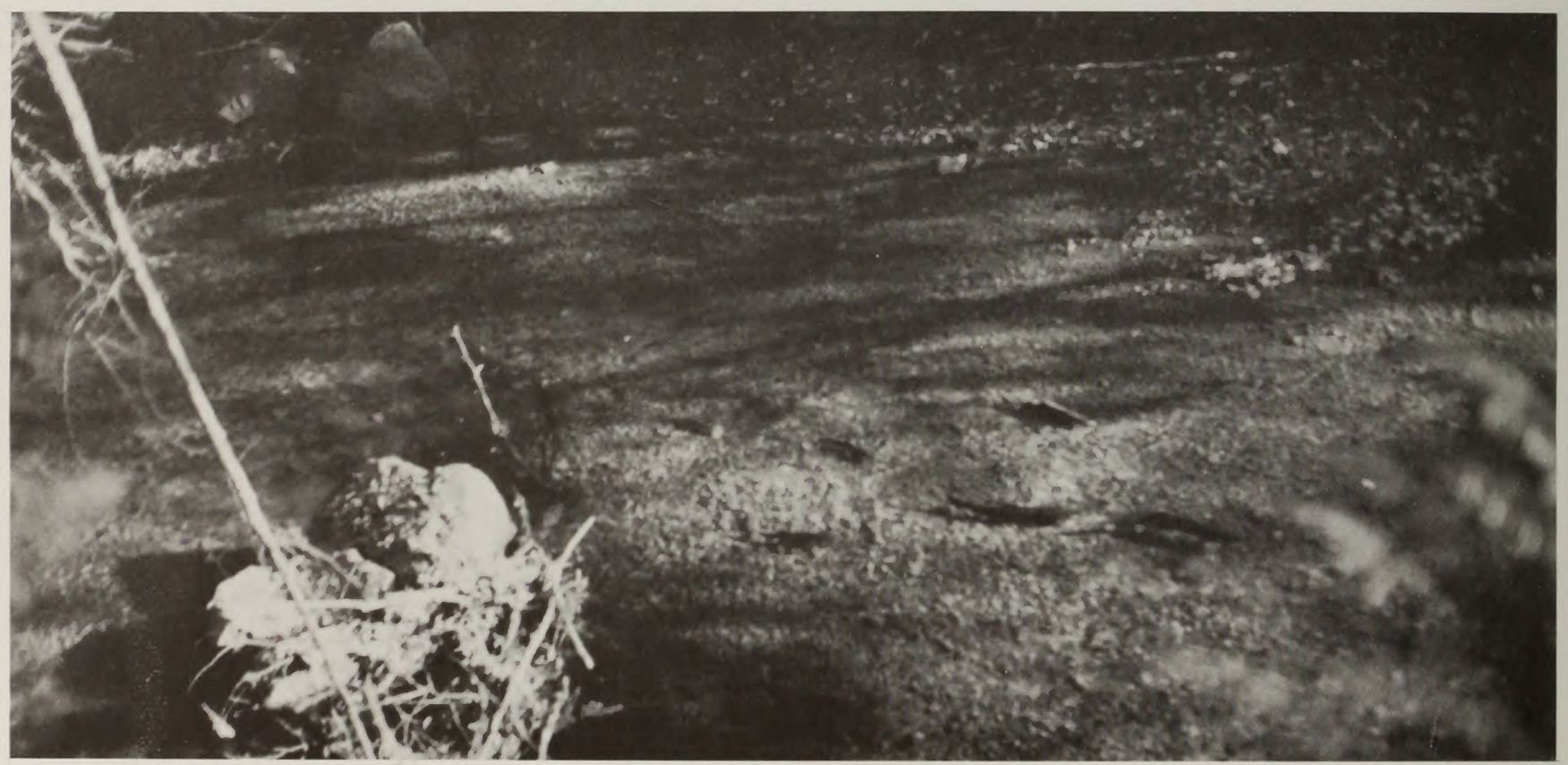

Spawning chinook salmon on a redd located about 60 feet upstream from the gabion shown in the other photo. Note two groups of boulders placed in Tioga Creek. Boulders placed in the middle of the stream create a small pool for resting. The boulders placed along the bank of Tioga Creek prevent erosion around the upper part of the gabion.

Cover Photo: John Anderson (on left), Coos Bay District Fishery Biologist, and John Crawford, former Chief of the Wildlife Division, Washington Office, discuss how gabion structures function to improve spawning and rearing habitat in Tioga Creek, tributary to the South Coos River, Coos County, Oregon. An aquatic habitat management plan developed under the authority of the Sikes Act was implemented by a Memorandum of Understanding between Menasha Corporation, the Oregon Department of Fish and Wildlife and the Bureau of Land Management during May 1982 to protect and enhance wild stocks of anadromous fish. To date, the Bureau has constructed $\mathbf{3 8}$ gabion structures (like the gabion Anderson and Crawford are standing on) and 7 boulder berms in Tioga Creek and tributaries to increase production of wild fish. 




\section{A Five-Year Comprehensive Anadromous Fish Habitat Enhancement Plan for Oregon Coastal Rivers}

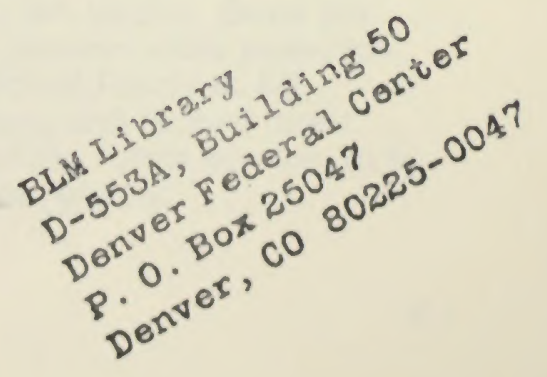





\section{Introduction and Purpose}

This report was prepared to provide current information concerning opportunities to improve the present productivity of anadromous salmonid habitat (primarily salmon and steelhead) on Bureau lands in coastal rivers of Oregon. Habitat rehabilitation and/or enhancement work is done to increase populations of wild fish, which results in greater numbers of fish available for harvest by recreational and commercial fisheries important to Oregon's coastal economy, communities and populace in general.

The proposed habitat projects listed in this report constitute a logical plan for orderly fish habitat development work by identified district priorities over a five-year period. If implemented, these projects would be closely coordinated with similar habitat work of the Oregon Department of Fish and Wildlife (ODFW), U.S. Forest Service and other concerned agencies and organizations to obtain maximum efficiency and benefits.

\section{Background}

The principal management responsibility of the BLM concerning fish production in streams on Bureau lands is to manage habitat to maintain highly-productive conditions for wild populations of fish. The Oregon Department of Fish and Wildlife is responsible for managing all fish populations within state waters. A good working relationship exists between the two agencies that was initially fostered by several appropriate memoranda of understanding. Many cooperative endeavors between the two agencies relating to salmon and steelhead management activities have occurred over the past 20 years.

Bureau land ownership in western Oregon is basically a "checkerboard" pattern due primarily to the revested 0\&C lands (refer to map). Because of this, the Bureau's "sphere of influence" extends to intermingled private and state lands as well as adjacent national forests. For this reason, fish habitat management activities on Bureau lands have also been closely coordinated with the Forest Service, local conservation organizations and private landowners. Numerous cooperative habitat projects to benefit wild fish runs have been completed with other organizations in past years. However, the full benefits possible from some projects on BLM lands were not realized on other "upstream" lands because complementary work on private lands was not accomplished, often due to lack of funds. Money appropriated to the Bureau can be spent only for projects on BLM lands.

At present there are 985 miles of anadromous fish habitat on BLM land in coastal streams of Oregon. Fish produced on Bureau lands contribute significantly to ocean and freshwater fisheries, which in turn provide important economic and social benefits to coastal communities. It was estimated, using 1981 values, that the annual net economic value of all fish attributable to BLM lands in western Oregon was approximately $\$ 4.5$ million (374,100 activity days), of which $\$ 2.7$ million $(111,700$ activity days plus the commercial catch) was attributable to anadromous fish produced in streams on BLM lands.

The original productivity of most coastal rivers and tributary streams has been reduced substantially due to man's activities and land uses since the 1870's. Timber harvesting and associated activities have had the greatest overall adverse impacts on coastal streams. However, agricultural development, channel alterations in lower reaches of rivers by snagging and ditching, road building, mining, and diking, dredging and filling of estuaries have all contributed to reduced productivity of anadromous fish habitat.

Historically, there were many fallen, large old-growth trees (trunks or parts) in Oregon's coastal streams that created some of the best spawning and rearing areas for young salmon and steelhead. The significance of this naturally occurring large woody material (LWM) to anadromous fish productivity was not fully recognized until recent years. LWM in stream channels provides an instream structure that creates habitat diversity in the form of riffles, pools and cover which are used by fish differently at various stages of their lives (see photo section). Without some larger trees to hold bedload movement and create pools, stream beds of higher gradient streams are subject to severe erosion and scouring. As a result, spawning gravels, food production and rearing areas have been greatly reduced.

Winter floods can cause localized accumulations of LWM in the form of debris jams that block upstream migrations of adult anadromous fish. This was a major problem when extensive logging occurred coastwide from the late 1940's until the early 1970's and streams were overloaded with logging debris. A substantial amount of money was spent removing logging debris during those years. In some streams "overcleaning" of naturally-occurring LWM occurred and widespread flooding scoured stream channels that eliminated pools and spawning gravels, contributing to the present lack of LWM and reduced capability of many streams to produce large numbers of anadromous fish.

The major reasons for reduced productivity of streams have been recognized. There is an increased awareness of fisheries and watershed values, and corrective actions have been taken in many instances to protect fish habitat. Since the mid 1970's streams have received more protection due to the 1971 Oregon Forest Practices Act, different land use allocations and revised forest management guidelines of the Forest Service, BLM and Oregon Department of Forestry. 
Considerable effort has been expended in the last 30 years by appropriate state and federal resource agencies and other organizations to maintain and increase natural production of anadromous fish in coastal rivers. As previously discussed, stream clearance of logging debris to maintain fish production in historic areas was the major activity. Providing fish passage at natural waterfalls by constructing fishways or blasting jump pools was another type of project that was commonly done to obtain production from formerly inaccessible areas. Relatively few instream habitat enhancement projects were done prior to 1970 because log jam removal and fish passage projects were considered highest priority.

The Bureau has had an active fish habitat improvement program for the last 15 years. The program has progressed from primarily log/debris jam clearance work and fish passage projects to more instream construction to improve or create spawning and rearing areas. The greatest amount of habitat work was done during FY 1981 when about $\$ 350,000$ was spent for 34 projects to improve habitat conditions and increase subsequent fish production in 50 miles of coastal streams. Because of this past work, relatively few log jam or small waterfall passage projects remain to be done on Bureau lands.

\section{Experience gained since 1970 has resulted in} successful instream construction work as verified by monitoring to evaluate the physical and biological results of the projects. Improved habitat conditions have resulted in increased numbers of young and adult fish. Photos to illustrate different types of completed projects are shown in the photo section. A steeppass fishway, conventional step-and-pool fish ladder and a waterfall that was blasted are examples of small fish passage projects. Different types of instream structures such as gabions, log sills and boulder beams are intended to produce desirable spawning and rearing conditions similar to those created naturally by large fallen trees and other LWM. Photos of a large rearing pool blasted in bedrock and a log jam removal project are also included.

\section{Present Situation}

Stream inventories, low catches by coastal fisheries and poor returns of adult spawning coho salmon indicate coastal streams are still producing far below full capability due to various factors limiting production. The ocean fisheries caught an unusually high percentage of coho populations during several years that also effected subsequent production. The adverse effects of "EI Nino" on ocean survival of coho salmon for several years are well documented and were another major factor in the low numbers of coho salmon in recent years. To protect these small coho populations, ocean fishing seasons were drastically curtailed during the last two years resulting in severe economic losses to coastal communities.

Land use plans of western Oregon districts call for increasing production of wild anadromous fish to help meet demand. Analyses in these plans show positive social and environmental benefits if wild fish production is increased. Improved habitat management through land use allocations and practices and habitat development projects are costeffective methods for increasing production of wild fish. These methods and identified habitat improvement opportunities are included in current land use plans.

In June 1982 the Oregon Fish and Wildlife Commission adopted a "Comprehensive Plan for Production and Management of Oregon's Anadromous Salmon and Trout, Part 1. General Considerations and Part 2. Coho Salmon Plan." One of the cornerstones of the coho salmon plan is to restore wild coho populations to optimum levels to maintain desired levels of long-term productivity and harvest. Habitat improvement work proposed in this five-year plan would help achieve management objectives for wild fish in the coho salmon plan and complement policies for all wild salmon and steelhead listed in Oregon Administrative Rules of the ODFW.

As previously discussed, considerable work was done in past years to rehabilitate or restore the productivity of coastal streams. The majority of the work was related to upstream fish passage, either at waterfalls, dams or in hundreds of miles of streams that were clogged with logging debris. Although the objectives of this work were accomplished, habitat deterioration has continued to occur in some streams due to some of man's activities and natural events such as floods. At the same time, some habitat conditions in other streams severely damaged in past years have improved, e.g. lower water temperatures due to more stream shading by riparian vegetation and bank stability. However, adequate amounts of important instream habitat components such as rearing pools, cover or spawning gravel are still lacking in many streams. These streams have little chance to recover their former productivity for many years without rehabilitation efforts.

Those factors still "limiting" fish production in streams on Bureau lands are shown in Table 1. Also shown are the types of projects that would correct those habitat limitations. The Bureau now has the personnel capability and experience in each district to do the necessary projects, provided a small number of temporary employees are hired to assist in constructing the projects. Many of the projects are labor intensive and would require close supervision to carefully install instream structures that will function properly and create the desired 
habitat conditions. Most of the larger projects would be accomplished by contracts.

Congressional funding was provided to the Bureau in FY 1985 to do survey and design and project planning work in coastal rivers. This work is now being accomplished so that important anadromous fish habitat projects can be completed as soon as funds are provided.

\section{Goal and Objectives}

The goal of the anadromous fish habitat enhancement plan is to provide and enhance the fishery potential of coastal streams in order to further contribute to the economic stability of the coastal communities and to the recreational and commercial fishing industries. In conformance with the O\&C Act of August 28, 1937, which requires management of the O\&C timber lands for timber production, protecting watersheds, and regulating stream flows and the requirements of the Federal Land Policy and Management Act of October 21, 1976 to manage on the basis of multiple use and sustained yield, the fish production capability of streams will be enhanced to more fully achieve their contribution to the multiple use benefits derived from these lands.

The objectives of the five-year plan are to:

- Increase habitat productivity in 985 miles of coastal streams on Bureau lands currently utilized by anadromous fish but producing below potential.

- Increase the amount of habitat available for producing anadromous fish by completing fish passage projects where feasible.

- Develop interagency habitat plans for coastal watersheds on a priority basis as agreed by concerned agencies and major landowners, e.g., use of "Coordinated Resource Management and Planning" (CRMP) process and interagency reports.

- Obtain maximum benefits for dollars spent through coordinated and cooperative fish habitat management efforts with other agencies and organizations.

\section{Management Approach}

Productivity of anadromous fish habitat on Bureau lands will be increased by:

- Implementing those decisions made in existing land use plans that benefit fish production, e.g, land use allocations, use of measures or practices designed to mitigate adverse effects on fish habitat from other activities and operations and construction of habitat improvement projects. - Cooperating with all other appropriate agencies and organizations at the local level to develop coordinated approaches for managing and improving anadromous fish habitat, i.e., CRMPs, interagency reports.

- Completing habitat development projects identified in this report as funds become available over a five-year period. This work would result in a substantial increase in the numbers of anadromous fish produced in existing habitat $(158,300 \mathrm{sq}$. meters plus 24 miles of improved fish passage) as well as 153 miles of now inaccessible habitat above impassable fish barriers.

\section{Data Analyses and Habitat Development Opportunities}

\section{Limiting Factors and Proposed Projects}

Limiting factors to anadromous fish production in 74 coastal rivers and tributary streams identified from stream inventories are listed in table 1. A lack of adequate spawning and rearing habitats and limited instream structures composed primarily of LWM were the major habitat factors determined to be limiting production. The lack of instream structure is a major cause for poor spawning and rearing conditions in many streams. In addition, 20 streams have total or partial barriers to the upstream migrations of adult salmon and steelhead.

The type and number of rehabilitation and enhancement projects to correct habitat problems are also listed in table 1. Rehabilitation generally refers to projects designed to restore productivity, whereas enhancement generally refers to projects that increase productivity above original levels. The end result is greater habitat productivity and increased numbers of fish available to the fisheries. There are a total of 84 projects proposed for the 74 streams shown in table 1.

The majority of the proposed project work involves construction of instream structures $(2,956)$ and pools (169) mostly in medium to small tributary streams where most of the coho salmon and steelhead spawning and rearing occurs. The use of different types of instream structures is intended to "restructure" key sections of streams by imitating large fallen trees and other LWM that creates desired spawning and rearing conditions, i.e., gravel riffles and deep pools. Large permanent pools blasted in bedrock provide needed rearing areas where this type of habitat is a limiting factor. Thirtyone off channel developments would be completed in side channels where the right conditions occur to produce large numbers of young fish.

Three types of fish passage projects are planned; $18 \mathrm{log}$ jams, 9 dam or road culverts and 10 waterfalls. The log jam and dam/culvert projects are all relatively small but require some work to provide fish passage. Two of the waterfall passage projects are major efforts that require engineering feasibility and design studies. These are Lake Creek Falls which would open 100 miles to anadromous fish above Triangle Lake and South Fork Alsea River Falls which would make about 13 miles accessible to anadromous fish. 
Table 1. Limiting Factors and Proposed Rehabilitation and Enhancement Projects for Anadromous Fish in Oregon Coastal Rivers

Limiting Factors

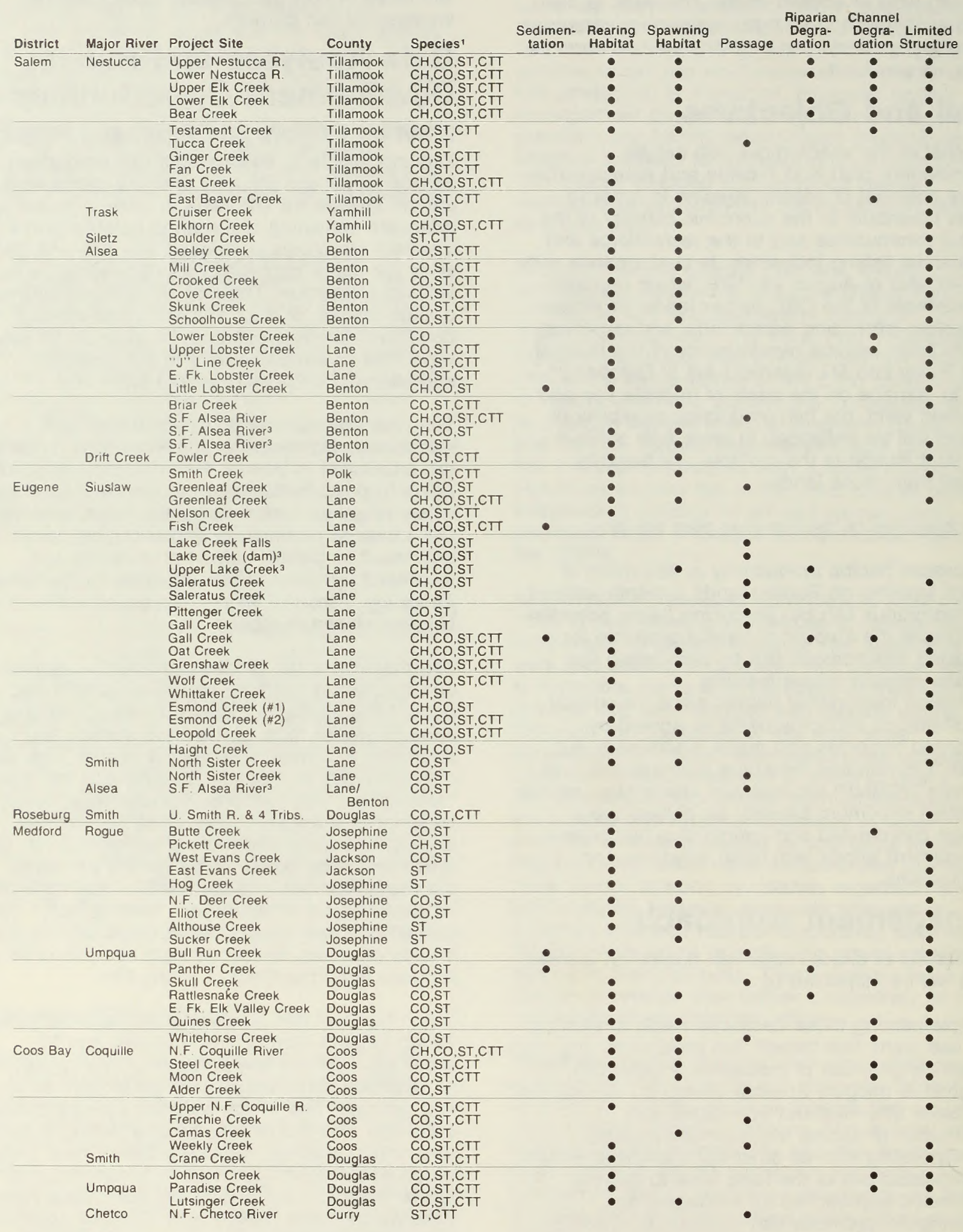

Key CH - Chinook, CO - Coho, ST - Steelhead, CTT - Sea Run Cutthroat Trout

2Instream structures include gabions, log sills, boulder berms, and loose trees that either fully or partially span the channel

aProjects to be done only if fish passage is provided first at downstream talls 


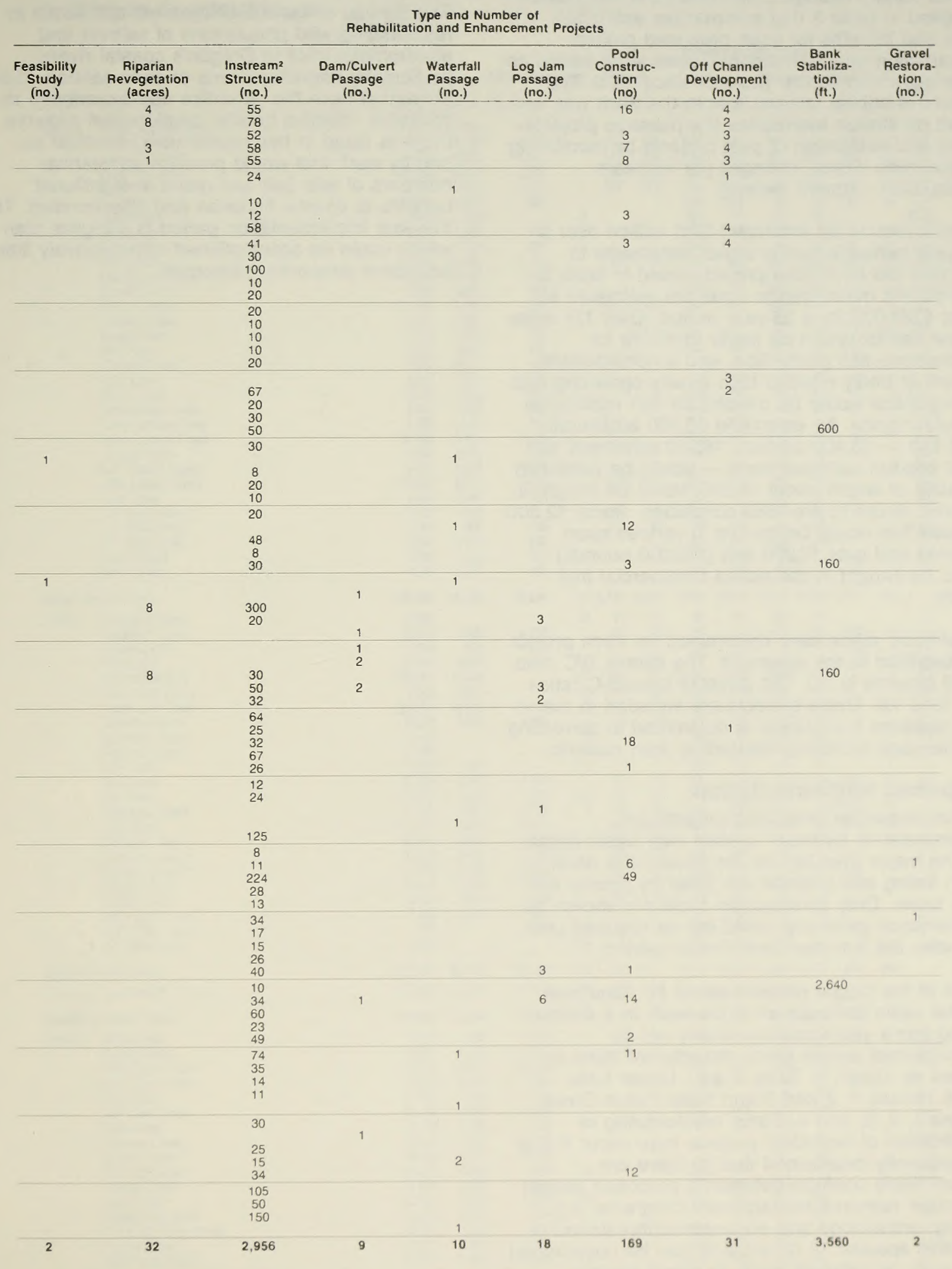

5 


\section{Estimated Costs and Benefits}

District fishery biologists submitted the information compiled in table 2 that summarizes estimated costs and benefits for each proposed project. Construction and maintenance costs were based on experience with similar projects since 1970 . The amount of habitat to benefit from the work was based on stream inventories (for passage projects miles) and evaluation of past projects by monitoring to determine habitat changes (for instream construction - square meters).

It would require an estimated $\$ 2.6$ million over a five-year period in nearly equal increments to complete the 84 habitat projects listed in table 2. Subsequent maintenance costs are estimated at about $\$ 281,000$ for a 25 -year period. Over 177 miles of new habitat would be made available for anadromous fish production, and a considerable amount of badly needed high quality spawning and rearing areas would be created for fish production on public lands. An estimated 53,200 additional adult fish $-33,400$ salmon, 16,300 steelhead and 3,500 sea-run cutthroat trout - would be produced annually, of which about 29,500 would be caught if fisheries return to pre-1983 conditions. About 12,300 of these fish would be caught in various sport fisheries and over 17,200 fish (126,000 pounds) would be caught in the ocean commercial troll fishery.

Benefit/cost ratios were determined for each project as described in the appendix. The overall $B / C$ ratio for all projects is 3.0. Two projects have $\mathrm{B} / \mathrm{C}$ ratios less than 1.0. These projects are included in the plan because the Bureau is committed to correcting fish passage problems created by road culverts.

\section{Proposed Implementation}

District personnel prioritized projects for implementation by major coastal river basin (table $3)$. The major river basins are shown in a northsouth listing and projects are listed by priority for each basin. Only construction costs are shown, as maintenance generally would not be required until well after the five-year construction period.

Some of the bigger projects. would be done over several years because all of the work in a district during some years could probably not be accomplished unless some projects are done in phases as shown in Table 3, e.g., Upper Lake Creek phases 1,2 and 3 and West Evans Creek phases $1,2,3$, and 4 . Some rescheduling or modification of individual projects may occur if it is subsequently determined that (1) there are irreconcilable conflicts between a proposed project and other resource management programs, e.g. mining, archeology and endangered/threatened or sensitive species, or (2) projects can be coordinated with work on adjacent lands to obtain greater benefits.

\section{Summary}

The Bureau of Land Management can assist in rejuvenating wild populations of salmon and anadromous trout in Oregon's coastal rivers, particularly depressed runs of coho salmon. Bureau personnel have the expertise and experience to construct effective habitat development projects. Projects listed in this report were identified as priority work that would produce substantial numbers of wild fish and result in significant benefits to coastal fisheries and communities. The five-year implementation period is a logical plan which could be accomplished with relatively little additional temporary personnel. 
Table 2 Estimated Costs and Benefits for Proposed Anadromous Fish Habitat Projects in Oregon Coastal Stream

\begin{tabular}{|c|c|c|c|c|c|c|c|c|c|c|c|c|c|c|}
\hline \multirow[b]{3}{*}{ District } & \multirow[b]{3}{*}{ Project Site } & \multirow{2}{*}{\multicolumn{2}{|c|}{$\operatorname{Costs}(\mathbf{S})$}} & \multirow{2}{*}{\multicolumn{2}{|c|}{ Habitat Benefitted }} & \multicolumn{8}{|c|}{ Annual Fish Production } & \multirow{3}{*}{$\begin{array}{r}B / C \\
\text { Ratio } \\
\end{array}$} \\
\hline & & & & & & \multicolumn{2}{|c|}{ Chinook } & \multicolumn{2}{|c|}{ Coho } & \multirow{2}{*}{\multicolumn{2}{|c|}{$\begin{array}{l}\text { Steelhead } \\
\text { Total Catch }\end{array}$}} & \multirow{2}{*}{\multicolumn{2}{|c|}{$\frac{\text { S.RCutthroat }}{\text { Total Catch }}$}} & \\
\hline & & Const. & Maint. & Sq. Meters & Miles & Total & Catch & Total & Catch & & & & & \\
\hline Salem & $\begin{array}{l}\text { Upper Nestucca River } \\
\text { Lower Nestucca River } \\
\text { Upper Elk Creek } \\
\text { Lower Elk Creek } \\
\text { Bear Creek } \\
\text { Testament Creek } \\
\text { Tucca Creek } \\
\text { Ginger Creek } \\
\text { Fan Creek } \\
\text { East Creek } \\
\text { East Beaver Creek } \\
\text { Cruiser Creek } \\
\text { Elkhorn Creek } \\
\text { Boulder Creek } \\
\text { Seeley Creek } \\
\text { Mill Creek } \\
\text { Crooked Creek } \\
\text { Cove Creek } \\
\text { Skunk Creek } \\
\text { Schoolhouse Creek } \\
\text { Lower Lobster Creek } \\
\text { Upper Lobster Creek } \\
\text { J' Line Creek } \\
\text { E.F. Lobster Creek } \\
\text { Little Lobster Creek } \\
\text { Briar Creek } \\
\text { S.F. Alsea River } \\
\text { S.F. Alsear River } \\
\text { S.F. Alsea River } \\
\text { Fowler Creek } \\
\text { Smith Creek }\end{array}$ & $\begin{array}{r}35,300 \\
49,500 \\
34,650 \\
39,450 \\
43,950 \\
13,750 \\
3,000 \\
5,800 \\
7,500 \\
40,300 \\
30,850 \\
7,200 \\
21,000 \\
3,000 \\
4,200 \\
4,200 \\
3,000 \\
3,000 \\
3,000 \\
3,200 \\
7,200 \\
30,000 \\
6,000 \\
9,000 \\
16,800 \\
5,400 \\
32,500 \\
2,400 \\
18,000 \\
3,000 \\
4,200 \\
\end{array}$ & $\begin{array}{r}5,900 \\
8,000 \\
5,500 \\
6,200 \\
5,800 \\
2,500 \\
300 \\
1,000 \\
1,200 \\
6,200 \\
4,500 \\
3,000 \\
10,000 \\
1,000 \\
2,000 \\
2,000 \\
1,000 \\
1,000 \\
1,000 \\
2,000 \\
3,000 \\
5,900 \\
2,000 \\
3,000 \\
5,000 \\
3,000 \\
1,000 \\
800 \\
2,000 \\
1,000 \\
2,000\end{array}$ & $\begin{array}{r}1,984 \\
3,330 \\
1,545 \\
1,933 \\
1,254 \\
263 \\
112 \\
187 \\
1,400 \\
1,215 \\
490 \\
326 \\
263 \\
526 \\
526 \\
263 \\
263 \\
263 \\
163 \\
1,200 \\
1,360 \\
326 \\
1,020 \\
680 \\
163 \\
210 \\
232 \\
263 \\
526 \\
\end{array}$ & 73 & $\begin{array}{l}128 \\
798 \\
454 \\
560 \\
557\end{array}$ & $\begin{array}{r}88 \\
535 \\
304 \\
375 \\
373\end{array}$ & $\begin{array}{r}794 \\
693 \\
534 \\
621 \\
578 \\
349 \\
103 \\
112 \\
154 \\
404 \\
447 \\
289 \\
722 \\
\\
216 \\
140 \\
140 \\
140 \\
140 \\
140 \\
236 \\
534 \\
217 \\
325 \\
280 \\
140 \\
628 \\
56 \\
280 \\
72 \\
140 \\
\end{array}$ & $\begin{array}{r}595 \\
520 \\
401 \\
466 \\
433 \\
249 \\
67 \\
90 \\
110 \\
303 \\
336 \\
217 \\
541 \\
\\
162 \\
105 \\
91 \\
91 \\
91 \\
91 \\
153 \\
401 \\
162 \\
244 \\
182 \\
91 \\
408 \\
40 \\
180 \\
54 \\
105\end{array}$ & $\begin{array}{r}440 \\
384 \\
592 \\
344 \\
278 \\
80 \\
163 \\
36 \\
43 \\
464 \\
208 \\
160 \\
400 \\
80 \\
120 \\
120 \\
80 \\
80 \\
80 \\
80 \\
\\
296 \\
80 \\
240 \\
160 \\
80 \\
210 \\
64 \\
160 \\
80 \\
80\end{array}$ & $\begin{array}{r}154 \\
134 \\
207 \\
120 \\
114 \\
39 \\
73 \\
9 \\
12 \\
162 \\
73 \\
56 \\
140 \\
26 \\
40 \\
40 \\
26 \\
26 \\
26 \\
26 \\
98 \\
96 \\
26 \\
79 \\
53 \\
26 \\
69 \\
21 \\
53 \\
26 \\
26\end{array}$ & $\begin{array}{r}243 \\
266 \\
202 \\
248 \\
118 \\
35 \\
16 \\
22 \\
188 \\
158 \\
2 \\
5 \\
2 \\
2 \\
2 \\
2 \\
2 \\
2 \\
2 \\
\\
9 \\
2 \\
4 \\
2 \\
178\end{array}$ & $\begin{array}{r}61 \\
67 \\
49 \\
63 \\
30 \\
10 \\
\\
4 \\
6 \\
47 \\
40 \\
1 \\
2 \\
1 \\
1 \\
1 \\
1 \\
1 \\
1 \\
1 \\
\\
3 \\
1 \\
2 \\
\\
1 \\
43 \\
\end{array}$ & $\begin{array}{r}6.3 \\
5.9 \\
8.0 \\
6.1 \\
5.2 \\
4.7 \\
20.8 \\
3.5 \\
3.4 \\
4.5 \\
3.4 \\
9.1 \\
13.7 \\
5.9 \\
11.2 \\
9.5 \\
10.1 \\
10.1 \\
10.1 \\
8.6 \\
2.8 \\
4.3 \\
6.7 \\
9.7 \\
6.9 \\
5.2 \\
5 \\
14.4 \\
3.7 \\
8.3 \\
7.3 \\
\end{array}$ \\
\hline \multicolumn{2}{|c|}{ Salem District Totals } & 490,350 & 98,800 & 22,286 & 8.3 & 4,194 & 2,812 & 9,624 & 6,979 & 5,682 & 1,980 & 1,716 & 439 & \\
\hline Eugene & $\begin{array}{l}\text { Greenleaf Creek } \\
\text { Greenleaf Creek } \\
\text { Nelson Creek } \\
\text { Fish Creek } \\
\text { Lake Creek Falls } \\
\text { Lake Creek (Dam) } \\
\text { Upper Lake Creek } \\
\text { Saleratus Creek } \\
\text { Saleratus Creek } \\
\text { Pittenger Creek } \\
\text { Gall Creek } \\
\text { Gall Creek } \\
\text { Oat Creek } \\
\text { Grenshaw Creek } \\
\text { Wolf Creek } \\
\text { Whittaker Creek } \\
\text { Esmond Creek } \# 1 \\
\text { Esmond Creek } \# 2 \\
\text { Leopold Creek } \\
\text { Haight Creek } \\
\text { North Sister Creek } \\
\text { North Sister Creek } \\
\text { S.F. Alsea Piver }\end{array}$ & $\begin{array}{r}17,500 \\
65,000 \\
2,000 \\
32,500 \\
250,000 \\
2,000 \\
300,000 \\
20,500 \\
3,000 \\
10,000 \\
7,500 \\
32,500 \\
57,000 \\
62,500 \\
125,000 \\
83,000 \\
88,500 \\
52,500 \\
43,000 \\
24,000 \\
7,000 \\
700 \\
3,600\end{array}$ & $\begin{array}{r}4,800 \\
800 \\
3,000 \\
12,500 \\
2,000 \\
30,000 \\
2,000 \\
\\
\\
100 \\
3,000 \\
5,200 \\
3,200 \\
6,400 \\
2,600 \\
3,200 \\
2,700 \\
2,600 \\
1,200 \\
2,400\end{array}$ & $\begin{array}{r}1,500 \\
1,700 \\
2,000 \\
6,000 \\
6,050 \\
4,400 \\
2,800 \\
6,500 \\
720 \\
750\end{array}$ & $\begin{array}{r}3 \\
1 \\
25 \\
35 \\
2\end{array}$ & $\begin{array}{r}20 \\
30 \\
20 \\
600 \\
378 \\
416 \\
60 \\
132 \\
56\end{array}$ & $\begin{array}{r}15 \\
20 \\
15 \\
450 \\
189 \\
278 \\
40 \\
89 \\
28\end{array}$ & $\begin{array}{r}60 \\
100 \\
20 \\
121 \\
2,000 \\
500 \\
2,000 \\
80 \\
60 \\
20 \\
100 \\
150 \\
300 \\
260 \\
300 \\
144 \\
156 \\
170 \\
79 \\
60 \\
30 \\
100\end{array}$ & $\begin{array}{r}45 \\
74 \\
15 \\
90 \\
1,488 \\
370 \\
1,400 \\
59 \\
44 \\
15 \\
74 \\
111 \\
222 \\
192 \\
222 \\
108 \\
117 \\
128 \\
59 \\
44 \\
22 \\
75\end{array}$ & $\begin{array}{r}250 \\
400 \\
10 \\
90 \\
1,000 \\
200 \\
1,000 \\
45 \\
50 \\
15 \\
40 \\
50 \\
160 \\
160 \\
200 \\
200 \\
128 \\
96 \\
104 \\
48 \\
40 \\
30 \\
40\end{array}$ & $\begin{array}{r}75 \\
120 \\
3 \\
24 \\
300 \\
60 \\
300 \\
15 \\
15 \\
5 \\
12 \\
15 \\
48 \\
48 \\
60 \\
66 \\
42 \\
32 \\
34 \\
16 \\
12 \\
9 \\
12\end{array}$ & $\begin{array}{l}200 \\
100 \\
100 \\
200\end{array}$ & $\begin{array}{l}40 \\
20 \\
20 \\
40\end{array}$ & $\begin{array}{r}3.7 \\
1.7 \\
2.2 \\
1.8 \\
2.6 \\
42.8 \\
1.7 \\
1.1 \\
7.1 \\
0.7 \\
3.5 \\
1.1 \\
1.3 \\
1.1 \\
1.5 \\
1.1 \\
1.2 \\
1.0 \\
1.5 \\
1.1 \\
2.0 \\
16.8 \\
7.6\end{array}$ \\
\hline
\end{tabular}

Eugene District Totals

Roseburg Up. Smith A. \& Tribs

Roseburg District Totals

Medord Butte Creek

Pickett Creek

West Evans Creek

East Evans Creek

Hog Creek

NF. Deer Creek

Elliot Creek

Althouse Creek

Sucker Creek

Bull Run Creek

Panther Cree

Skull Creek

Rattlesnake Creek

E. Fk. Elk Valley Creek

Quines Creek

Whitehorse Creek

Medford District Totals
$1,289,800 \quad 87,700$

$137.500 \quad 12,500$

$137,500 \quad 12,500$

$5.600 \quad 800$

$12.800 \quad 1.100$

$118,800 \quad 13,500$

$19.600 \quad 2,800$

$10,400 \quad 1,300$

$22,400 \quad 3,400$

$8.700 \quad 1.500$

$19.500 \quad 1,500$

$32,500 \quad 2.500$

$21,500 \quad 4,000$

$24,800 \quad 1,000$

$29,000 \quad 3.400$

$32.925 \quad 6.000$

$14.100 \quad 2,300$

$30,300 \quad 4,900$

$62.550 \quad 7,400$

$465,475 \quad 57,400$
60,620

17,500

17,500

200
1.070

1,350
11,350

868
211

1,326

405

1,475
4900

4,900
655

$\begin{array}{rr}655 & 1.5 \\ 200 & \\ 1.085 & 1.6 \\ 975 & \end{array}$

975
705

1,570
2.234

29,229 $\begin{array}{lllllllll}52 & 3,055 & 2,111 & 6,810 & 4,974 & 4,356 & 1,323 & 1,070 & 206\end{array}$

$\begin{array}{lllllll}684 & 506 & 375 & 112 & 36 & 6 & 1.2\end{array}$

$$
\begin{array}{lllllllll}
0 & 0 & 0 & 684 & 506 & 375 & 112 & 36 & 6
\end{array}
$$$$
\begin{array}{llllll}
74 & 50 & 47 & 35 & 32 & 10 \\
& & & 88 & 34
\end{array}
$$$$
\begin{array}{rrrr}
\quad & 176 & 52 \\
& & 59 & 27
\end{array}
$$$$
\begin{array}{rrrr}
295 & 219 & 197 & 59 \\
89 & 66 & 60 & 18
\end{array}
$$

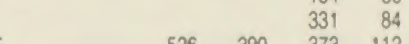$$
\begin{array}{llll}
526 & 390 & 373 & 112 \\
193 & 142 & 168 & 51
\end{array}
$$

$\begin{array}{lllll}737 & 546 \quad 637 & 190\end{array}$

$\begin{array}{llll}771 & 571 & 514 & 155\end{array}$

$\begin{array}{llll}245 & 182 & 164 & 49\end{array}$

$\begin{array}{rlll}548 & 405 & 367 & 110\end{array}$

$\begin{array}{rrrrrrr}1.9 & & & 1,022 & 719 & 736 & 221 \\ 5 & 74 & 50 & 5,311 & 3,895 & 4,651 & 1,398\end{array}$

37
17
2.2
18
2.6
2.8
17
1.1
7.1
0.7
3.5
1.1
3
1.1
15
1.1
2
10
1.5
1.1
20
6.8
76

2.2
2.8
1.8
1.9
1.9
3.4
2.6
2.2
19
64
24
76
5.9
44
46
4.3


Table 2 Estimated Costs and Benefits for Proposed Anadromous Fish Habitat Projects in Oregon Coastal Stream (continued)

\begin{tabular}{|c|c|c|c|c|c|c|c|c|c|c|c|c|c|c|}
\hline \multirow[b]{3}{*}{ District } & \multirow[b]{3}{*}{ Project Site } & & & \multirow{2}{*}{\multicolumn{2}{|c|}{ Habitat Benefitted }} & \multicolumn{8}{|c|}{ Annual Fish Production } & \multirow{3}{*}{$\begin{array}{r}B / C \\
\text { Ratio }\end{array}$} \\
\hline & & \multicolumn{2}{|c|}{ Costs $(\$)$} & & & \multicolumn{4}{|c|}{ Chinook } & \multirow{2}{*}{\multicolumn{2}{|c|}{$\begin{array}{l}\text { Steelhead } \\
\text { Total Catch }\end{array}$}} & \multirow{2}{*}{\multicolumn{2}{|c|}{$\begin{array}{l}\text { S.RLutthroat } \\
\text { Total Catch }\end{array}$}} & \\
\hline & & Const. & Maint. & Sq. Meters & Miles & Total & Catch & Total & Catch & & & & & \\
\hline Coos Bay & $\begin{array}{l}\text { N.F. Coquille River } \\
\text { Steel Creek } \\
\text { Moon Creek } \\
\text { Alder Creek } \\
\text { Upper N.F.Coquille River } \\
\text { Frenchie Creek } \\
\text { Camas Creek } \\
\text { Weekly Creek } \\
\text { Crane Creek } \\
\text { Johnson Creek } \\
\text { Paradise Creek } \\
\text { Lutzinger Creek } \\
\text { N.F. Chetco River }\end{array}$ & $\begin{array}{r}56,000 \\
8,540 \\
6,000 \\
400 \\
6,600 \\
32,800 \\
12,600 \\
7,400 \\
15,840 \\
15,000 \\
45,000 \\
17,000 \\
10,000\end{array}$ & $\begin{array}{r}3,500 \\
1,400 \\
1,100 \\
\\
800 \\
2,000 \\
2,500 \\
500 \\
3,400 \\
1,500 \\
5,000 \\
2,500 \\
500\end{array}$ & $\begin{array}{r}5,529 \\
1,429 \\
789 \\
1,858 \\
5,463 \\
\\
2,104 \\
3,249 \\
4,900 \\
3,344\end{array}$ & $\begin{array}{r}0.25 \\
1.5 \\
1\end{array}$ & 1,985 & 1,191 & $\begin{array}{r}141 \\
92 \\
50 \\
26 \\
376 \\
98 \\
115 \\
90 \\
112 \\
98 \\
328 \\
164\end{array}$ & $\begin{array}{r}93 \\
69 \\
37 \\
17 \\
248 \\
74 \\
76 \\
67 \\
84 \\
74 \\
246 \\
123\end{array}$ & $\begin{array}{r}11 \\
84 \\
40 \\
10 \\
5 \\
60 \\
100 \\
40 \\
68 \\
120 \\
200 \\
100 \\
400\end{array}$ & $\begin{array}{r}4 \\
28 \\
12 \\
3 \\
2 \\
2 \\
18 \\
30 \\
12 \\
20 \\
36 \\
60 \\
30 \\
100\end{array}$ & $\begin{array}{r}6 \\
50 \\
\\
30 \\
25 \\
100 \\
70 \\
65 \\
400\end{array}$ & $\begin{array}{r}1 \\
8 \\
\\
5 \\
4 \\
16 \\
14 \\
12 \\
50\end{array}$ & $\begin{array}{r}4.9 \\
3.6 \\
2.3 \\
15.5 \\
5.8 \\
0.9 \\
2.6 \\
3.4 \\
1.7 \\
2.6 \\
1.8 \\
2.4 \\
6.7\end{array}$ \\
\hline Coos Bay & District Totals & 233,180 & 24,700 & 28,665 & 11.75 & 1,985 & 1,191 & 1,690 & 1,208 & 1,238 & 355 & 750 & 110 & \\
\hline Grand Tot & lals & $2,616,305$ & 281,100 & 158,300 & 177.05 & 9,308 & 6,164 & 24,119 & 17,562 & 16,302 & 5,168 & 3,572 & 761 & 3.0 \\
\hline
\end{tabular}


Table 3 Estimated Construction Costs and Benefits for Proposed Anadromous Fish Habitat Projects by Major River Basin

\begin{tabular}{|c|c|c|c|c|c|c|c|c|c|c|c|}
\hline \multirow[b]{3}{*}{ River Basin and Project Sile } & \multirow{3}{*}{$\begin{array}{l}\text { Cons1. } \\
\text { Cosis }\end{array}$} & \multirow{2}{*}{\multicolumn{2}{|c|}{ abita1 Benefilted }} & \multicolumn{8}{|c|}{ Annual Fish Production } \\
\hline & & & & Chin & & \multicolumn{2}{|c|}{ Coho } & \multicolumn{2}{|c|}{ Sleelhead } & \multicolumn{2}{|c|}{ S.RCutthroal } \\
\hline & & Sq. Meters & Miles & Total & Catch & Tolal & Catch & Total & Catch & Total & Caich \\
\hline $\begin{array}{l}\text { Trask } \\
\text { Cruiser Creek } \\
\text { Elkhorn Creek }\end{array}$ & $\begin{array}{r}\$ 7,200 \\
\$ 21,000\end{array}$ & $\begin{array}{l}490 \\
326\end{array}$ & & 1,012 & 676 & $\begin{array}{l}289 \\
722 \\
\end{array}$ & $\begin{array}{l}217 \\
541\end{array}$ & $\begin{array}{l}160 \\
400\end{array}$ & $\begin{array}{r}56 \\
140 \\
\end{array}$ & $\begin{array}{l}2 \\
5\end{array}$ & $\begin{array}{l}1 \\
2 \\
\end{array}$ \\
\hline Tolal & $\$ 28,200$ & 816 & 0 & 1,012 & 678 & 1,011 & 758 & 560 & 196 & 7 & 3 \\
\hline $\begin{array}{l}\text { Nestucca } \\
\text { Upper Nestucca River } \\
\text { Lower Elk Creek } \\
\text { Tucca Creek } \\
\text { Lower Nestucca River } \\
\text { Upper Elk Creek } \\
\text { Bear Creek } \\
\text { Fan Creek } \\
\text { Testamenl Creek } \\
\text { Ginger Creek } \\
\text { East Beaver Creek } \\
\text { East Creek }\end{array}$ & $\begin{array}{r}\$ 35,300 \\
\$ 39,450 \\
\$ 3,000 \\
\$ 49,500 \\
\$ 34,650 \\
\$ 43,950 \\
\$ 7,500 \\
\$ 13,750 \\
\$ 5,800 \\
\$ 30,850 \\
\$ 40,300\end{array}$ & $\begin{array}{r}1,984 \\
1,933 \\
\\
3,330 \\
1,545 \\
1,254 \\
187 \\
263 \\
112 \\
1,215 \\
1,400 \\
\end{array}$ & 1 & $\begin{array}{l}128 \\
560 \\
798 \\
454 \\
557\end{array}$ & $\begin{array}{r}88 \\
375 \\
535 \\
304 \\
373\end{array}$ & $\begin{array}{l}794 \\
621 \\
103 \\
693 \\
534 \\
578 \\
154 \\
349 \\
112 \\
447 \\
404\end{array}$ & $\begin{array}{r}595 \\
466 \\
67 \\
520 \\
401 \\
433 \\
110 \\
249 \\
90 \\
336 \\
303 \\
\end{array}$ & $\begin{array}{r}440 \\
344 \\
163 \\
384 \\
592 \\
278 \\
43 \\
80 \\
36 \\
208 \\
464\end{array}$ & $\begin{array}{r}154 \\
120 \\
73 \\
134 \\
207 \\
114 \\
12 \\
39 \\
9 \\
73 \\
162 \\
\end{array}$ & $\begin{array}{r}243 \\
248 \\
\\
266 \\
202 \\
118 \\
22 \\
35 \\
16 \\
158 \\
188 \\
\end{array}$ & $\begin{array}{r}61 \\
63 \\
\\
67 \\
49 \\
30 \\
6 \\
10 \\
4 \\
40 \\
47\end{array}$ \\
\hline Tolal & $\$ 304,050$ & 13,223 & 1 & 2,595 & 1,741 & 4,789 & 3,570 & 3,032 & 1,097 & 1,496 & 377 \\
\hline
\end{tabular}

\begin{tabular}{|c|c|c|c|c|c|c|c|c|c|c|}
\hline $\begin{array}{l}\text { Siletz } \\
\text { Boulder Creek } \\
\text { Fowler Creek } \\
\text { Smith Creek }\end{array}$ & $\begin{array}{l}\$ 3,000 \\
\$ 3,000 \\
\$ 4,200 \\
\end{array}$ & $\begin{array}{l}263 \\
263 \\
526\end{array}$ & & & & $\begin{array}{r}72 \\
140\end{array}$ & $\begin{array}{r}54 \\
105\end{array}$ & $\begin{array}{l}80 \\
80 \\
80\end{array}$ & $\begin{array}{l}26 \\
26 \\
26\end{array}$ & $\begin{array}{l}2 \\
2 \\
2\end{array}$ \\
\hline $\begin{array}{l}\text { Tolal } \\
\text { Alsea } \\
\text { Little Lobster Creek } \\
\text { Briar Creek } \\
\text { S.F. Alsea River } \\
\text { Lower Lobsier Creek } \\
\text { Upper Lobster Creek } \\
\text { J' Line Creek } \\
\text { E.F. Lobster Creek } \\
\text { Seeley Creek } \\
\text { Mill Creek } \\
\text { Crooked Creek } \\
\text { Cove Creek } \\
\text { Skunk Creek } \\
\text { Schoolhouse Creek } \\
\text { S.F. Alsea River } \\
\text { S.F. Alsea River } \\
\text { S.F. Alsea River }\end{array}$ & $\begin{array}{r}\$ 10,200 \\
\$ 16,800 \\
\$ 5,400 \\
\$ 32,500 \\
\$ 7,200 \\
\$ 30,000 \\
\$ 6,000 \\
\$ 9,000 \\
\$ 4,200 \\
\$ 4,200 \\
\$ 3,000 \\
\$ 3,000 \\
\$ 3,000 \\
\$ 3,200 \\
\$ 18,000 \\
\$ 3,600 \\
\$ 2,400\end{array}$ & $\begin{array}{r}1,052 \\
\\
680 \\
163 \\
1,200 \\
1,360 \\
326 \\
1,020 \\
526 \\
526 \\
263 \\
263 \\
263 \\
163 \\
232 \\
210 \\
\end{array}$ & 5.5 & $\begin{array}{l}405 \\
70\end{array}$ & $\begin{array}{r}271 \\
47\end{array}$ & $\begin{array}{r}280 \\
140 \\
628 \\
236 \\
534 \\
217 \\
325 \\
216 \\
140 \\
140 \\
140 \\
140 \\
140 \\
280 \\
100 \\
56\end{array}$ & $\begin{array}{r}182 \\
91 \\
408 \\
153 \\
401 \\
162 \\
244 \\
162 \\
105 \\
91 \\
91 \\
91 \\
91 \\
180 \\
75 \\
40\end{array}$ & $\begin{array}{r}240 \\
\\
160 \\
80 \\
210 \\
\\
296 \\
80 \\
240 \\
120 \\
120 \\
80 \\
80 \\
80 \\
80 \\
160 \\
40 \\
64 \\
\end{array}$ & $\begin{array}{l}78 \\
\\
53 \\
26 \\
69 \\
\\
98 \\
26 \\
79 \\
40 \\
40 \\
26 \\
26 \\
26 \\
26 \\
53 \\
12 \\
21 \\
\end{array}$ & $\begin{array}{l}9 \\
2 \\
4 \\
2 \\
2 \\
2 \\
2 \\
2 \\
2\end{array}$ \\
\hline Tolal & $\$ 151,500$ & 7,195 & 12.8 & 587 & 393 & 3,712 & 2,567 & 1,890 & 621 & 207 \\
\hline $\begin{array}{l}\text { Siuslaw } \\
\text { Greenleaf Creek } \\
\text { Greenleaf Creek } \\
\text { Nelson Creek } \\
\text { Fish Creek } \\
\text { Gall Creek } \\
\text { Gall Creek } \\
\text { Grenshaw Creek } \\
\text { Lake Creek Falls } \\
\text { Lake Creek (Dam) } \\
\text { Saleratus Creek } \\
\text { Saleratus Creek } \\
\text { Wolf Creek } \\
\text {-Up. Lake Cr. } 1 \\
\text { Pittenger Creek } \\
\text { Oat Creek } \\
\text { Whittaker Creek } \\
\text {-Up. Lake Cr. } 2 \\
\text { Esmond Creek -1 } \\
\text { Esmond Creek -2 } \\
\text { Leopold Creek } \\
\text { - Up. Lake Cr. } 3 \\
\text { Haight Creek }\end{array}$ & $\begin{array}{r}\$ 17,500 \\
\$ 65,000 \\
\$ 2,000 \\
\$ 32,500 \\
\$ 7,500 \\
\$ 32,500 \\
\$ 62,500 \\
\$ 250,000 \\
\$ 2,500 \\
\$ 20,500 \\
\$ 3,000 \\
\$ 125,000 \\
\$ 50,000 \\
\$ 10,000 \\
\$ 57,000 \\
\$ 83,000 \\
\$ 50,000 \\
\$ 88,500 \\
\$ 52,500 \\
\$ 43,000 \\
\$ 200,000 \\
\$ 24,000\end{array}$ & $\begin{array}{r}4,500 \\
200 \\
1,500 \\
1,500 \\
2,000 \\
\\
\\
2,000 \\
\\
6,000 \\
3,333 \\
\\
1,700 \\
6,050 \\
3,333 \\
4,400 \\
2,800 \\
6,500 \\
13,334 \\
720\end{array}$ & $\begin{array}{r}2.5 \\
2 \\
100 \\
18 \\
3 \\
\\
1 \\
3.5\end{array}$ & $\begin{array}{r}34 \\
60 \\
\\
189 \\
\\
20 \\
20 \\
500 \\
50 \\
10 \\
\\
600 \\
83 \\
\\
30 \\
378 \\
83 \\
416 \\
60 \\
132 \\
334 \\
56 \\
\end{array}$ & $\begin{array}{r}25 \\
40 \\
127 \\
15 \\
15 \\
375 \\
38 \\
7 \\
\\
450 \\
62 \\
\\
20 \\
189 \\
63 \\
278 \\
40 \\
89 \\
250 \\
28\end{array}$ & $\begin{array}{r}60 \\
100 \\
20 \\
121 \\
100 \\
150 \\
260 \\
2,000 \\
500 \\
80 \\
60 \\
300 \\
333 \\
20 \\
300 \\
\\
333 \\
144 \\
156 \\
170 \\
1,334 \\
79\end{array}$ & $\begin{array}{r}45 \\
74 \\
15 \\
90 \\
74 \\
111 \\
192 \\
1,488 \\
370 \\
59 \\
44 \\
222 \\
233 \\
15 \\
222 \\
233 \\
108 \\
117 \\
128 \\
934 \\
59\end{array}$ & $\begin{array}{r}250 \\
400 \\
10 \\
90 \\
40 \\
50 \\
160 \\
1,000 \\
200 \\
45 \\
50 \\
200 \\
166 \\
15 \\
160 \\
200 \\
167 \\
128 \\
96 \\
104 \\
667 \\
48\end{array}$ & $\begin{array}{r}75 \\
120 \\
3 \\
24 \\
12 \\
15 \\
48 \\
300 \\
60 \\
15 \\
15 \\
60 \\
50 \\
5 \\
48 \\
66 \\
50 \\
42 \\
32 \\
34 \\
200 \\
16\end{array}$ & $\begin{array}{r}200 \\
20 \\
50 \\
\\
200 \\
100\end{array}$ \\
\hline Total & $\$ 1,278,500$ & 59,870 & 145 & 3,055 & 2,111 & 6,620 & 4,833 & 4,246 & 1,290 & 1,070 \\
\hline $\begin{array}{l}\text { Smith } \\
\text {-S. Fork \& Tribs. } \\
\text { - U.S.R \& Cleghorn } \\
\text { Johnson Creek } \\
\text { North Sister Creek } \\
\text { North Sister Creek }\end{array}$ & $\begin{array}{r}\$ 27,500 \\
\$ 27,500 \\
\$ 15,000 \\
\$ 7,000 \\
\$ 700\end{array}$ & $\begin{array}{r}3,500 \\
3,500 \\
3,249 \\
750\end{array}$ & 1.5 & & & $\begin{array}{r}137 \\
137 \\
98 \\
60 \\
30\end{array}$ & $\begin{array}{r}101 \\
102 \\
74 \\
44 \\
22\end{array}$ & $\begin{array}{r}75 \\
75 \\
120 \\
40 \\
30\end{array}$ & $\begin{array}{r}22 \\
23 \\
36 \\
12 \\
9\end{array}$ & $\begin{array}{r}? \\
8 \\
100\end{array}$ \\
\hline
\end{tabular}


Table 3 Estimated Construction Costs and Benefits for Proposed Anadromous Fish Habitat Projects by Major River Basin (continued)

\begin{tabular}{|c|c|c|c|c|c|c|c|c|c|c|c|}
\hline \multirow[b]{3}{*}{ River Basin and Project Site } & \multirow{3}{*}{$\begin{array}{l}\text { Const. } \\
\text { Costs }\end{array}$} & \multirow{2}{*}{\multicolumn{2}{|c|}{ bitat Benefitted }} & \multicolumn{8}{|c|}{ Annual Fish Production } \\
\hline & & & & Chine & & \multicolumn{2}{|c|}{ Coho } & \multicolumn{2}{|c|}{ Steelhead } & \multicolumn{2}{|c|}{ S.RCutthroat } \\
\hline & & Sq. Meters & Miles & Total & Catch & Total & Catch & Total & Catch & Total & Catch \\
\hline $\begin{array}{l}\text {-U.S.R. \& Halfway } \\
\text {-Up. Smith River } \\
\text { Crane Creek } \\
\text {-U.S.R. \& Summit }\end{array}$ & $\begin{array}{l}\$ 27,500 \\
\$ 27,500 \\
\$ 15,840 \\
\$ 27,500\end{array}$ & $\begin{array}{l}3,500 \\
3,500 \\
2,104 \\
3,500\end{array}$ & & & & $\begin{array}{l}137 \\
136 \\
112 \\
137\end{array}$ & $\begin{array}{r}101 \\
101 \\
84 \\
101\end{array}$ & $\begin{array}{l}75 \\
75 \\
68 \\
75\end{array}$ & $\begin{array}{l}23 \\
22 \\
20 \\
22\end{array}$ & $\begin{array}{r}7 \\
7 \\
25 \\
7\end{array}$ & $\begin{array}{l}1 \\
1 \\
4 \\
1\end{array}$ \\
\hline Total & $\$ 176,040$ & 23,603 & 1.5 & 0 & 0 & 984 & 730 & 633 & 189 & 161 & 26 \\
\hline $\begin{array}{l}\text { Umpqua } \\
\text {-Skull Cr.1 } \\
\text { - Rattlesnake Cr.1 } \\
\text { - Quines Creek } 1 \\
\text { Lutzinger Creek } \\
\text { - Whitehorse Cr.1 } \\
\text { - Bull Run Cr.1 } \\
\text { E. Fk. Elk Valley Cr. } \\
\text { Paradise Creek } \\
\text { Panther Creek } \\
\text { - Quines Creek } 2 \\
\text { - Bull Run Cr.2 } \\
\text { - Skull Cr.2 } \\
\text { - Rattlesnake Cr.2 } \\
\text { - Whitehorse Cr.2 }\end{array}$ & $\begin{array}{l}\$ 22,900 \\
\$ 16,900 \\
\$ 15,150 \\
\$ 17,000 \\
\$ 51,900 \\
\$ 10,750 \\
\$ 14,100 \\
\$ 45,000 \\
\$ 24,800 \\
\$ 15,150 \\
\$ 10,750 \\
\$ 6,100 \\
\$ 16,025 \\
\$ 10,650 \\
\end{array}$ & $\begin{array}{r}890 \\
505 \\
785 \\
3,344 \\
1,855 \\
330 \\
705 \\
4,900 \\
200 \\
785 \\
325 \\
195 \\
470 \\
379\end{array}$ & $\begin{array}{r}1.9 \\
0.75 \\
\end{array}$ & & & $\begin{array}{l}582 \\
396 \\
274 \\
164 \\
848 \\
263 \\
245 \\
328 \\
193 \\
274 \\
263 \\
155 \\
375 \\
174 \\
\end{array}$ & $\begin{array}{l}431 \\
293 \\
203 \\
123 \\
597 \\
195 \\
182 \\
246 \\
142 \\
202 \\
195 \\
115 \\
278 \\
122 \\
\end{array}$ & $\begin{array}{l}503 \\
264 \\
184 \\
100 \\
611 \\
186 \\
164 \\
200 \\
168 \\
183 \\
187 \\
134 \\
250 \\
125 \\
\end{array}$ & $\begin{array}{r}150 \\
80 \\
55 \\
30 \\
183 \\
56 \\
49 \\
60 \\
51 \\
55 \\
56 \\
40 \\
75 \\
38\end{array}$ & 65 & $\begin{array}{l}12 \\
14\end{array}$ \\
\hline Total & $\$ 277,175$ & 15,668 & 5 & 0 & 0 & 4,534 & 3,324 & 3,259 & 978 & 135 & 26 \\
\hline $\begin{array}{l}\text { Coquille } \\
\text { Moon Creek } \\
\text { Frenchie Creek } \\
\text { N.F. Coquille River } \\
\text { Camas Creek } \\
\text { Upper N.F. Coquille R } \\
\text { Weekly Creek } \\
\text { Alder Creek } \\
\text { Steel Creek }\end{array}$ & $\begin{array}{r}\$ 6,000 \\
\$ 32,800 \\
\$ 56,000 \\
\$ 12,600 \\
\$ 6,600 \\
\$ 7,400 \\
\$ 400 \\
\$ 8,540\end{array}$ & $\begin{array}{r}789 \\
5,529 \\
5,463 \\
1,858 \\
\\
1,429\end{array}$ & $\begin{array}{r}1.5 \\
\\
1 \\
0.25\end{array}$ & 1,985 & 1,191 & $\begin{array}{r}50 \\
98 \\
141 \\
115 \\
376 \\
90 \\
26 \\
92\end{array}$ & $\begin{array}{r}37 \\
74 \\
93 \\
76 \\
248 \\
67 \\
17 \\
69\end{array}$ & $\begin{array}{r}40 \\
60 \\
11 \\
100 \\
5 \\
40 \\
10 \\
84\end{array}$ & $\begin{array}{r}12 \\
18 \\
4 \\
30 \\
2 \\
2 \\
12 \\
3 \\
28\end{array}$ & $\begin{array}{r}1 \\
50 \\
3 \\
\\
6 \\
30\end{array}$ & $\begin{array}{l}0 \\
8 \\
0\end{array}$ \\
\hline $\begin{array}{l}\text { Total } \\
\text { Rog gue } \\
\text { N.F. Deer Creek } \\
\text { Elliot Creek } \\
\text { Pickett Creek } \\
\text {-W. Evans Cr. } 1 \\
\text { East Evans Creek } \\
\text { Hog Creek } \\
\text {-W. Evans Cr. } 2 \\
\text { Althouse Creek } \\
\text { Sucker Creek } \\
\text { Butte Creek } \\
- \text { W. Evans Cr. } 3 \\
\text {-W. Evans Cr. } 4\end{array}$ & $\begin{array}{r}\$ 22,400 \\
\$ 8,700 \\
\$ 12,800 \\
\$ 28,050 \\
\$ 19,600 \\
\$ 10,400 \\
\$ 34,750 \\
\$ 19,500 \\
\$ 32,500 \\
\$ 5,600 \\
\$ 32,150 \\
\$ 23,850\end{array}$ & $\begin{array}{r}1,326 \\
405 \\
1,070 \\
2,679 \\
868 \\
211 \\
3,360 \\
1,475 \\
4,900 \\
200 \\
3,030 \\
2,281\end{array}$ & 2.75 & 1,985 & 1,191 & $\begin{array}{r}295 \\
89 \\
\\
198 \\
\\
\\
248 \\
\\
\\
47 \\
224 \\
168\end{array}$ & $\begin{array}{r}219 \\
66 \\
\\
146 \\
\\
\\
184 \\
\\
\\
35 \\
165 \\
125\end{array}$ & $\begin{array}{r}197 \\
60 \\
88 \\
133 \\
176 \\
59 \\
167 \\
184 \\
331 \\
32 \\
151 \\
114\end{array}$ & $\begin{array}{l}59 \\
18 \\
34 \\
40 \\
52 \\
27 \\
50 \\
56 \\
84 \\
10 \\
46 \\
34\end{array}$ & 90 & 14 \\
\hline Total & $\$ 250,300$ & 21,805 & 0 & 74 & 50 & 1,269 & 940 & 1,692 & 510 & 0 & 0 \\
\hline $\begin{array}{l}\text { Chetco } \\
\text { N.F. Chetco River }\end{array}$ & $\$ 10,000$ & & 9 & & & & & 400 & 100 & 400 & 50 \\
\hline Grand Total & $\$ 2,616,305$ & 158,300 & 177.05 & 9,308 & 6,164 & 24,119 & 17,562 & 16,302 & 5,168 & 3,572 & 761 \\
\hline
\end{tabular}




\section{Appendix}

\section{Methods used to calculate fish production and catch and B/C Ratios}

For instream habitat projects, annual production of adult coho, steelhead and sea-run cutthroat trout was estimated by first determining how much rearing habitat would be improved and calculating the increase in young fish produced from available research studies and monitoring data. Then, survival estimates and average sport and commercial catch rates provided by the Oregon Department of Fish and Wildlife (ODFW) were used to calculate total adult fish produced and catch. In some cases where the amount of spawning habitat was also a limiting factor, the number of fish expected to subsequently return and use spawning habitat created by the project were also used in the analysis, i.e., (No. redds) $x$ (No. eggs per redd) $x$ (percent survival to adults) = (total adults produced or catch and escapement). For chinook salmon, survival data from the ODFW were used with the number of expected redds or spawning females for the project (based on monitoring of past BLM projects) to calculate adults produced. Catch was determined by applying average sport and commercial harvest rates for the fisheries to the total number of chinook produced.

A different method was used to calculate the numbers of adults produced and caught from fish passage projects. Long-term average fish-per-mile escapement values were multiplied by the miles of new spawning habitat that would become available by the project to obtain the expected spawning population. The number of females and subsequent egg potential were then calculated. Appropriate survival and harvest rates were then used to calculate the adults produced and catch.

Benefit/cost ratios were determined for each project in the State Office by a microcomputer program developed by the State Office economist. The ODFW provided current information needed for the analysis, i.e., ex-vessel price paid per pound to commercial fishermen, dressed weight of commercially caught fish, harvest rates and activity days per fish for different sport fisheries by species, and value per activity day (net willingness to pay) for different fisheries by species. The full ex-vessel value was used for the commercial catch to be representative of marginal additions to the catch of existing fishermen. Other studies have used 90 percent of ex-vessel price for periods of underemployment and 50 percent for periods of full employment of commercial fishing fleets.

An 8.375 percent discount rate and a 25-year project life were used in the analysis, although some passage and construction projects will last much longer, e.g., bedrock pool construction, log jam passage. A one-time maintenance cost was programmed for the twelfth year of most projects, based on the fact that most projects are types of instream construction that experience has shown will require some maintenance at about that time. Some fishways often require annual maintenance, but this is difficult to determine until the project has been functional for several years. The one-time maintenance cost was therefore also used for most fish passage projects.

Two types of benefit/cost program analyses were used; one for instream construction and the other for fish passage projects. For instream construction projects, it was assumed that spawning escapements would be adequate to provide sufficient numbers of young fish to occupy the newly created rearing habitat so that full credit was given to adult production during their first cycle of return but delayed an appropriate number of years based on maturity by species. For fish passage projects, the same general procedure was used except full credit for adult production was not given until the second cycle of return. It was assumed that full production in streams above barriers would not occur immediately because sufficient spawners would probably not be available to fully seed new production areas. For this reason, only one-half of expected adult production was used during the first cycle of return. 


\section{Large Woody Material (LWM) in Streams}

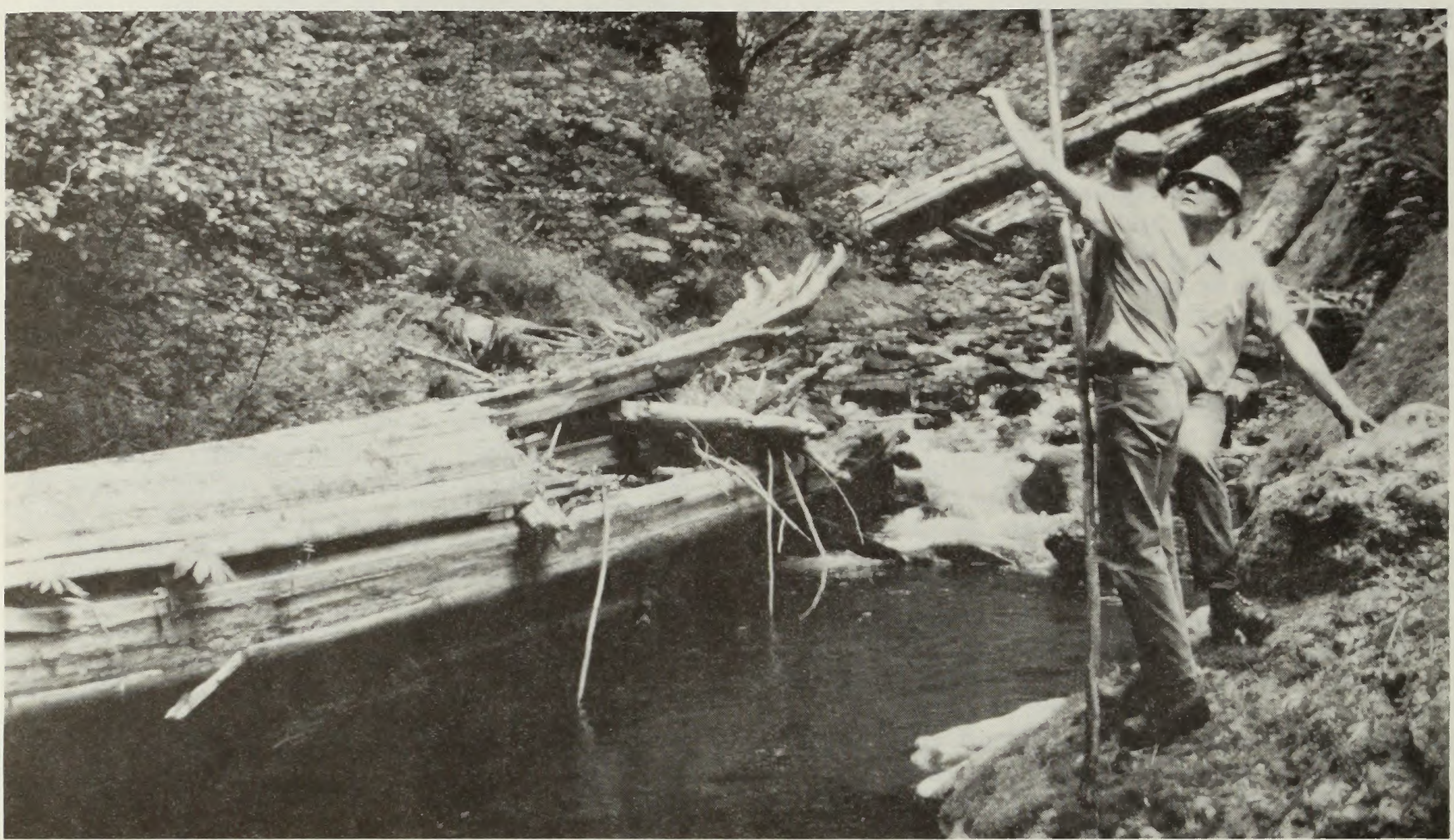

East Creek (Nestucca River) - natural occurring LWM provides instream structure that creates deep pools and cover for fish. Merle Marshall, now Area Manager, Salem District, and Dave Luman, now retired BLM Wildlife Biologist, discuss the importance of riparian vegetation to the productivity of streams.

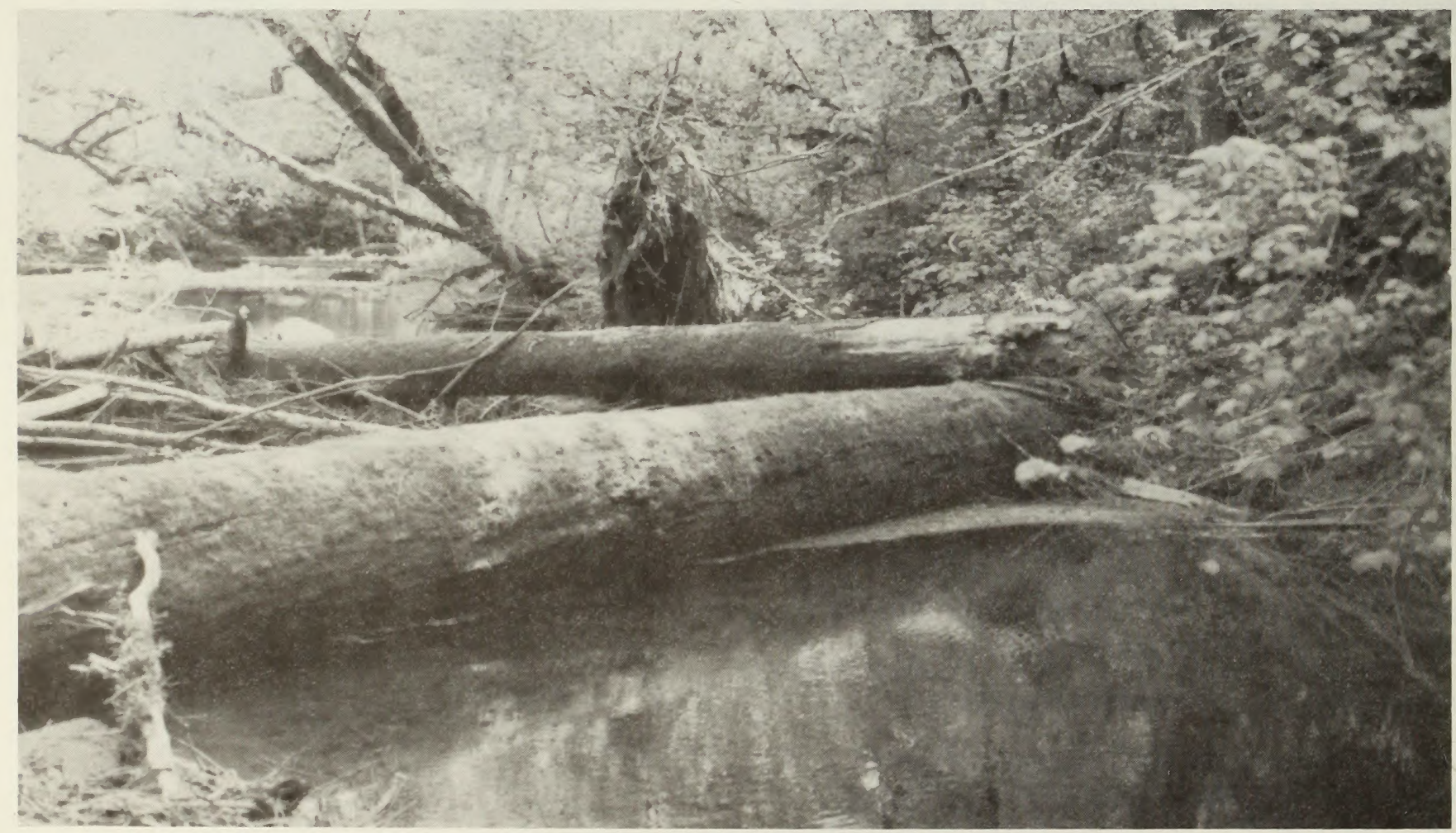

West Fork (Smith River) - loose accumulations of LWM create excellent rearing areas and cover for young fish and cover for adults before spawning. The large log will eventually settle into the streambed and create an upstream spawning riffle and downstream pool. 


\section{Fish Passage Projects}

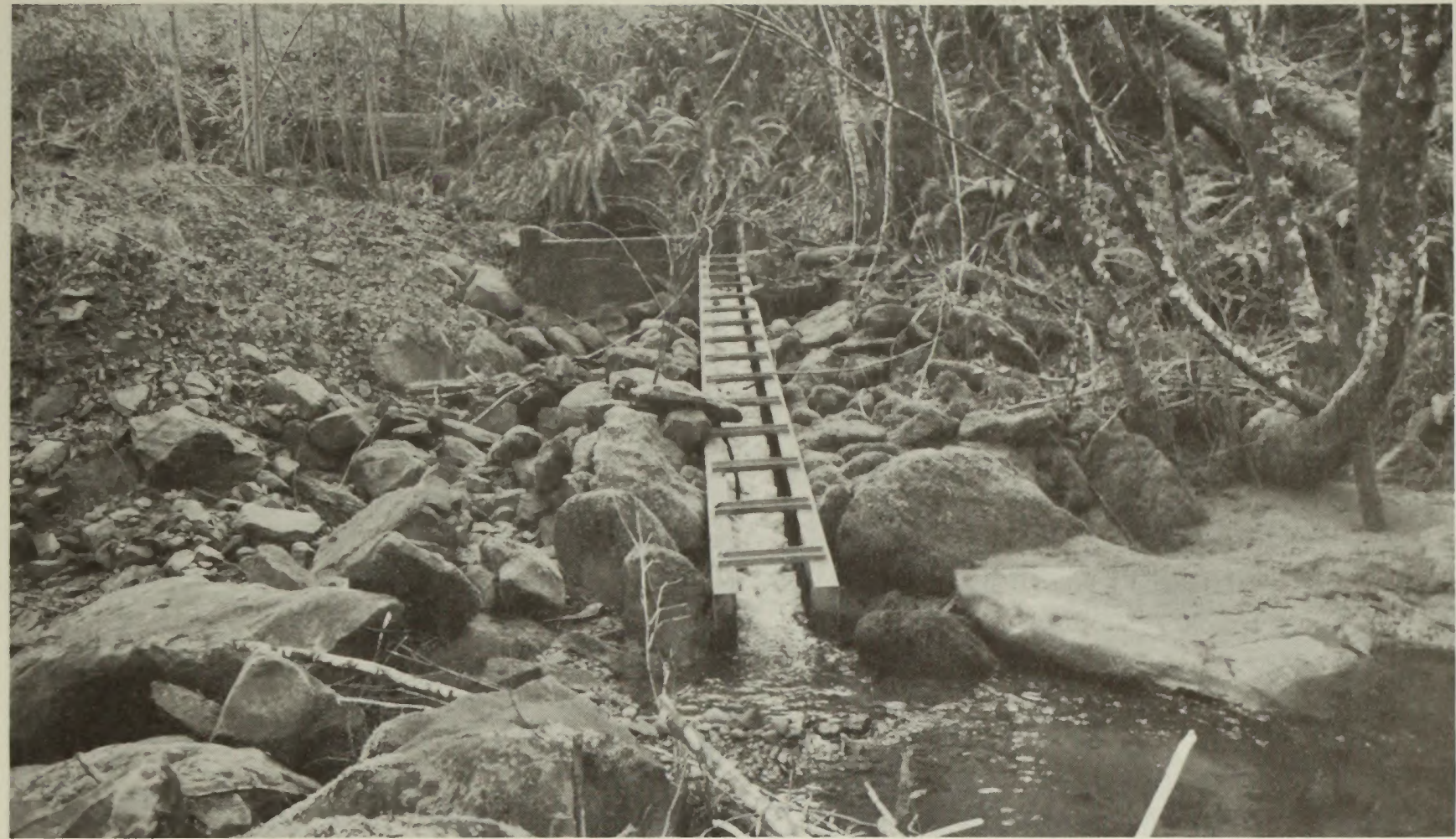

Vincent Creek (Smith River) - a small Alaska type steeppass fishway installed in a tributary stream to provide fish passage through a road culvert.

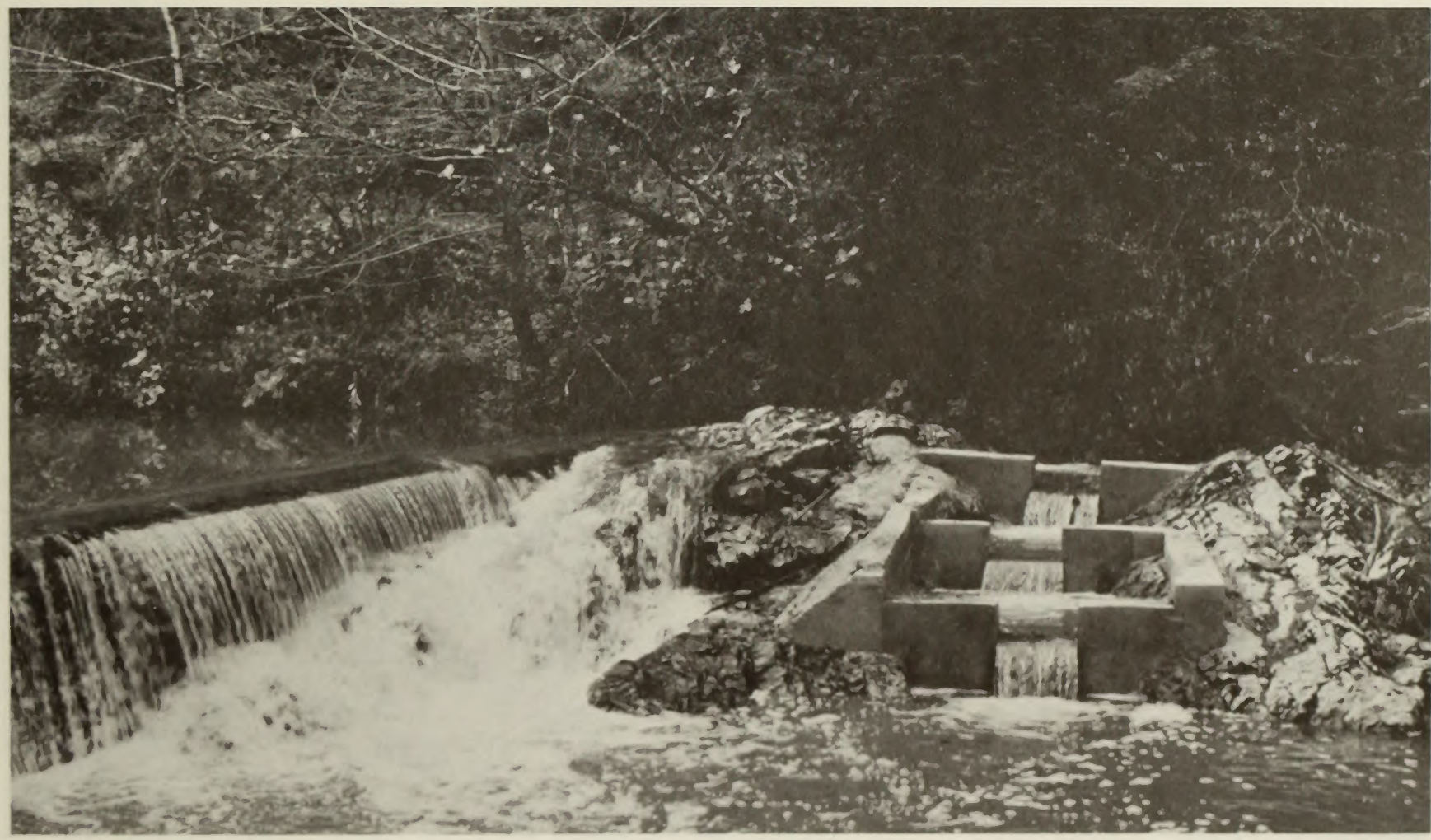

East Fork Evans Creek (Rogue River) - a smali, conventional concrete step-and-pool fishway constructed at a iow irrigation dam to provide anadromous fish easy access to upstream spawning areas at ail streamflows. 


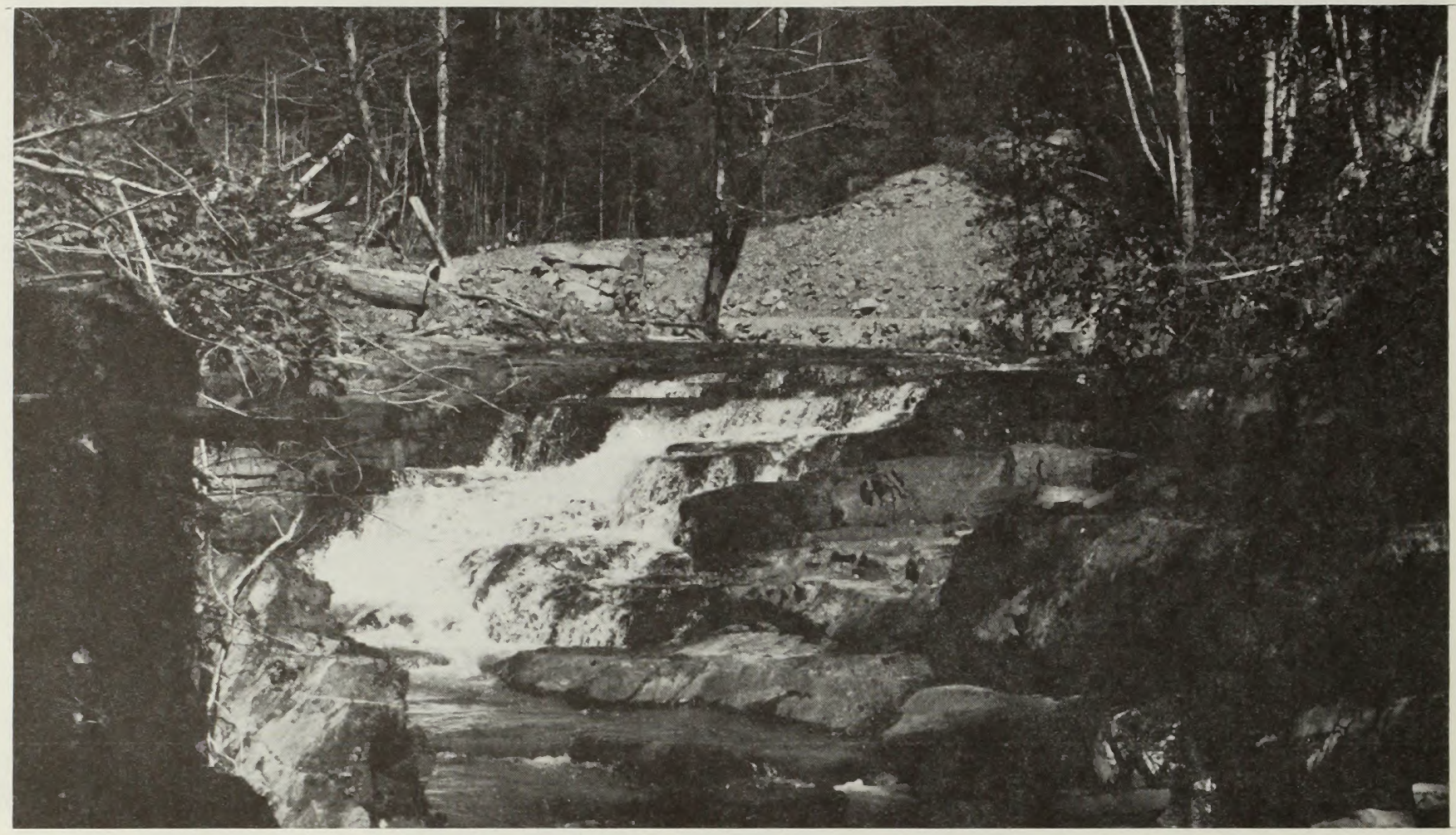

Little Wolf Creek Falls (Umpqua River) (before blasting) - this 14-foot waterfall was a total obstruction to the upstream migration of all anadromous fish.

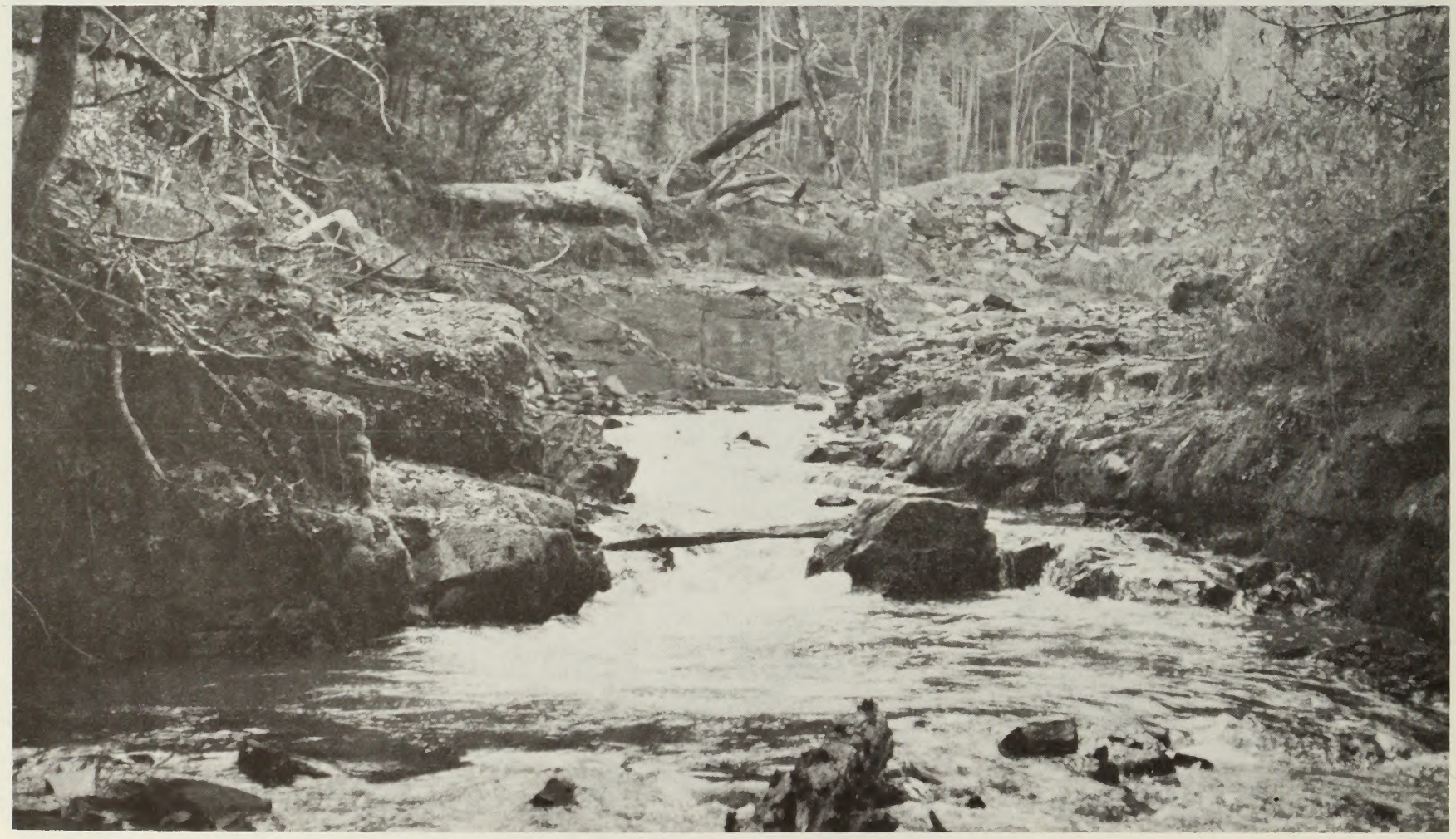

Little Wolf Creek Falls (after blasting) - this project made $41 / 2$ miles of excellent spawning and rearing area accessible for anadromous fish production. Nine small concrete fish weirs were subsequently built in the blasted channel to provide easy fish passage at all flows. 


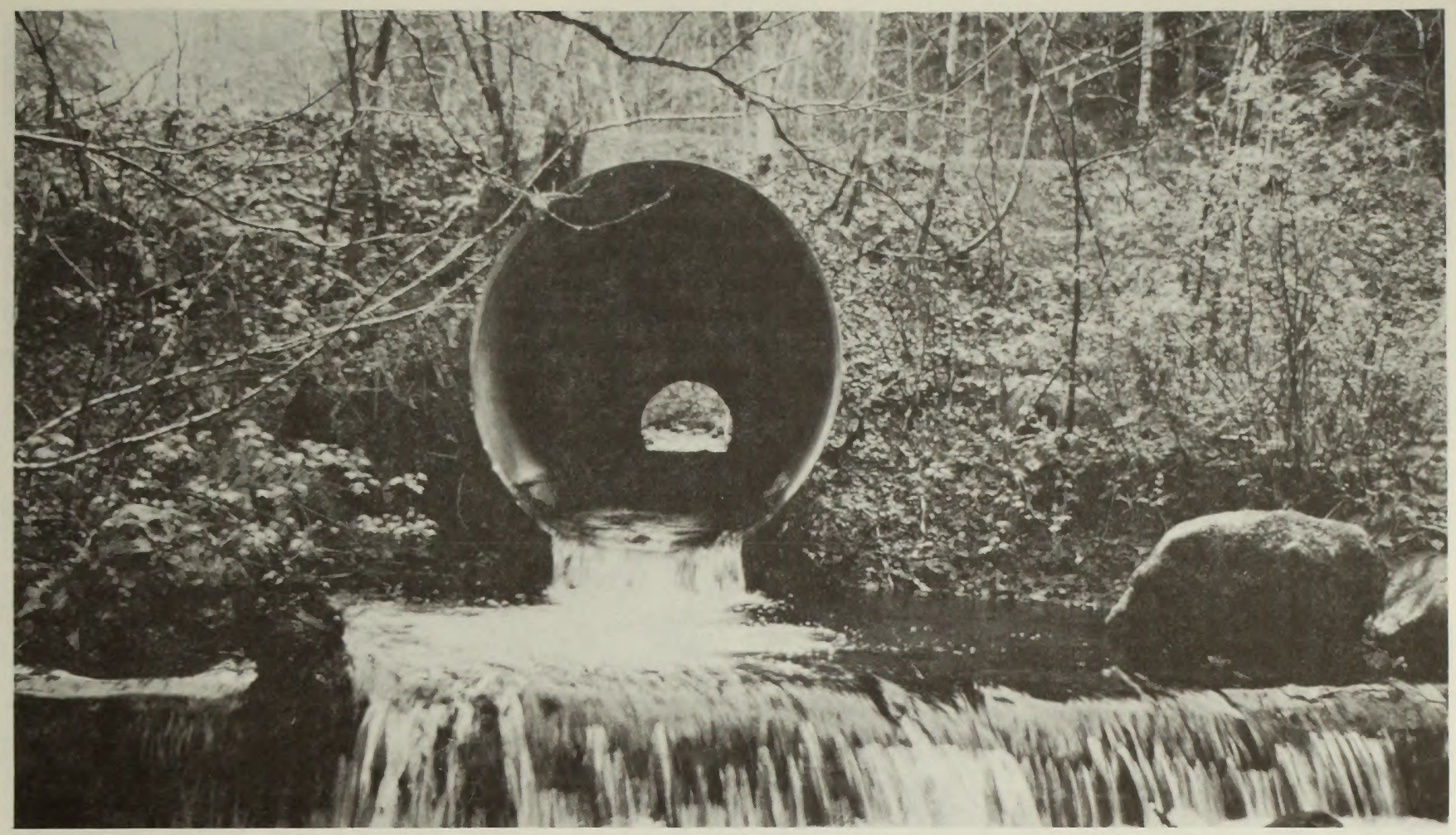

Fan Creek (Nestucca River) - a singie "log sill” installed to provide a jump pool and easy fish passage through a road culvert.

\section{Instream Construction Projects}

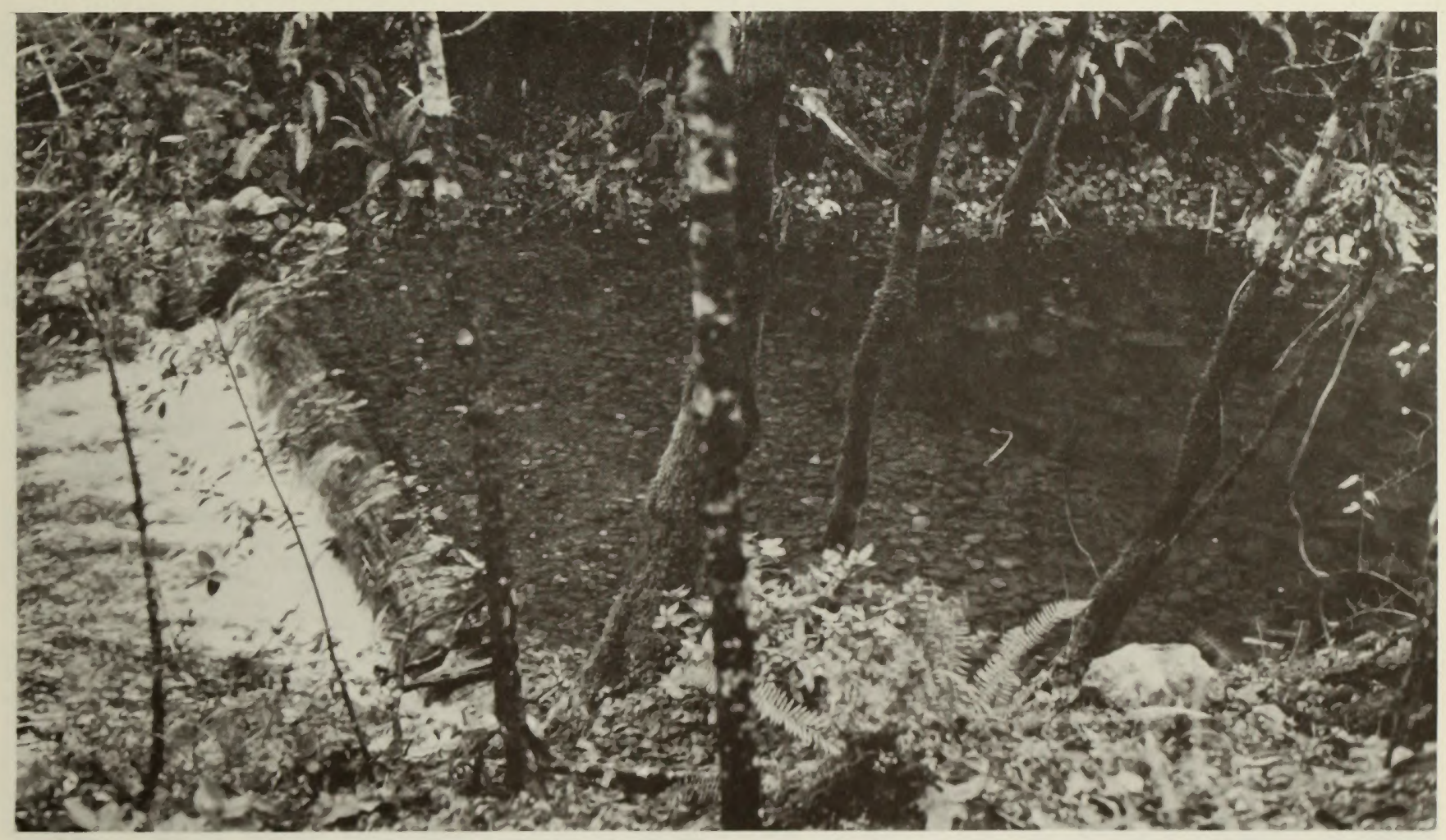

Pickett Creek (Rogue River) - a "log sill" spawning structure with spawning gravel added upstream that is utilized by both chinook saimon and steeihead trout. 


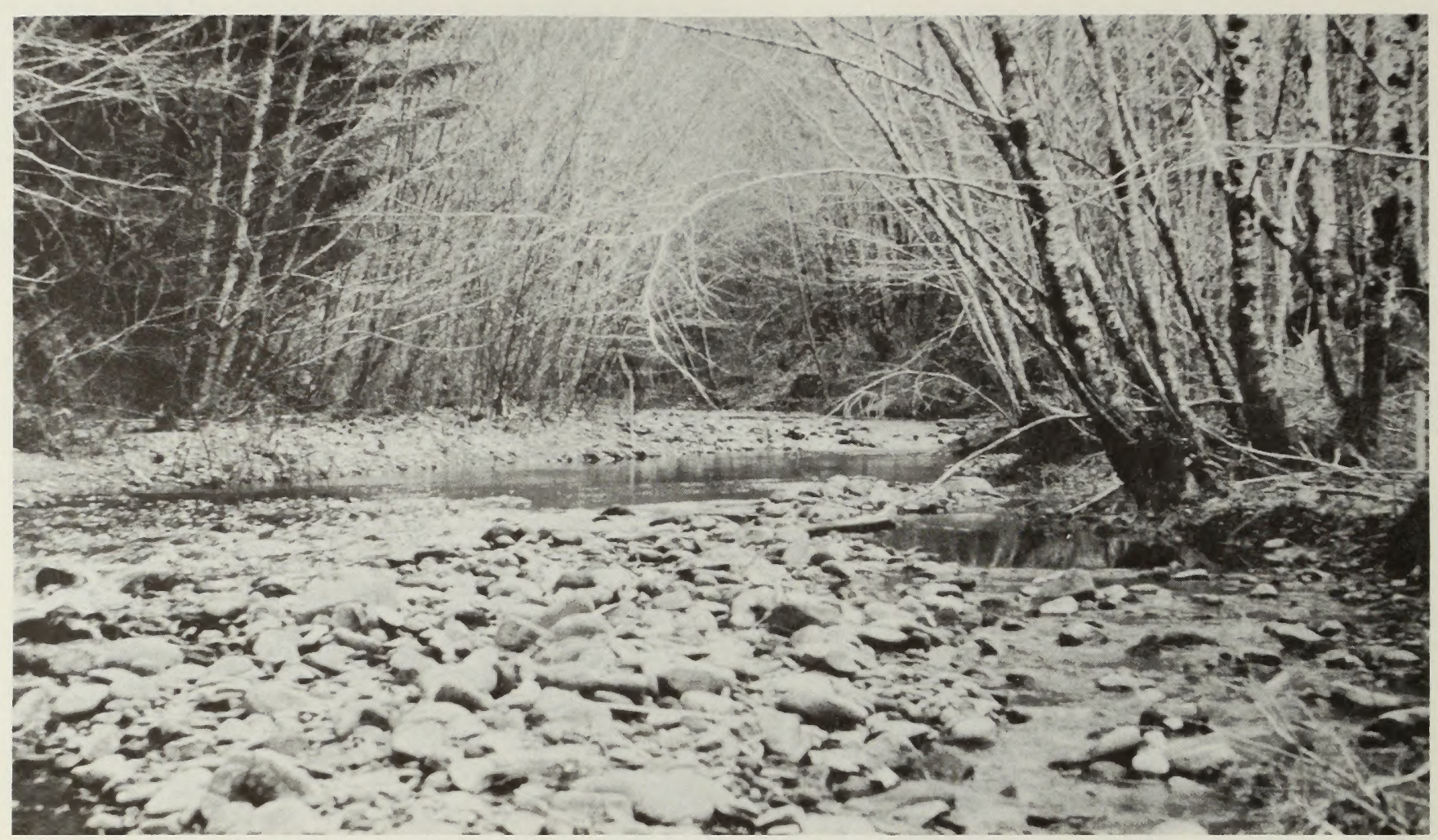

East Fork of Lobster Creek (Alsea River) - Photo of Gabion site taken before construction during early spring and decreasing stream flows.

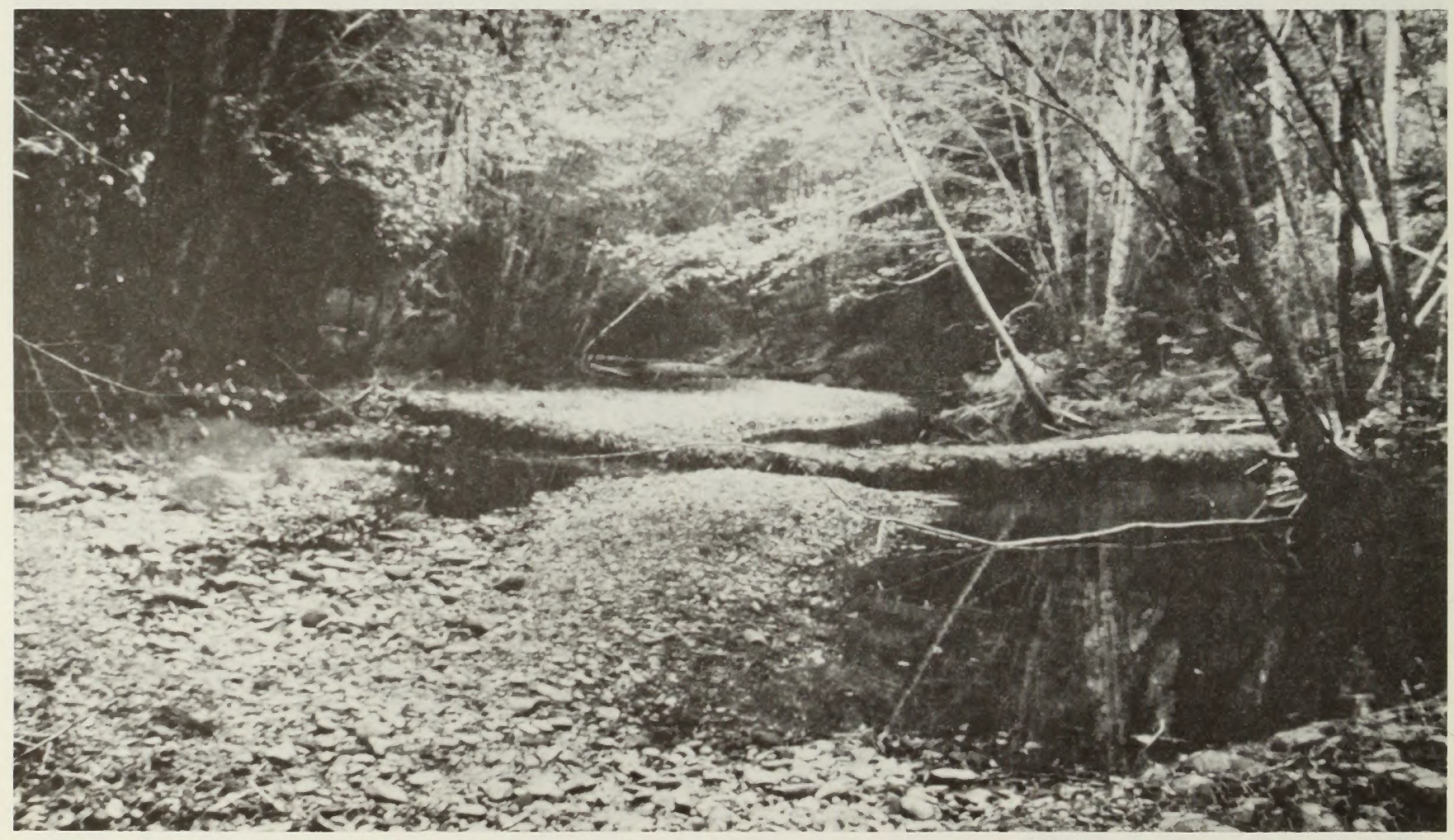

East Fork of Lobster Creek - Photo taken after construction of two standard " $V$ " wire rock gabions during summer low flow period. Note natural accretion of spawning gravel riffles and pools created by gabions. 


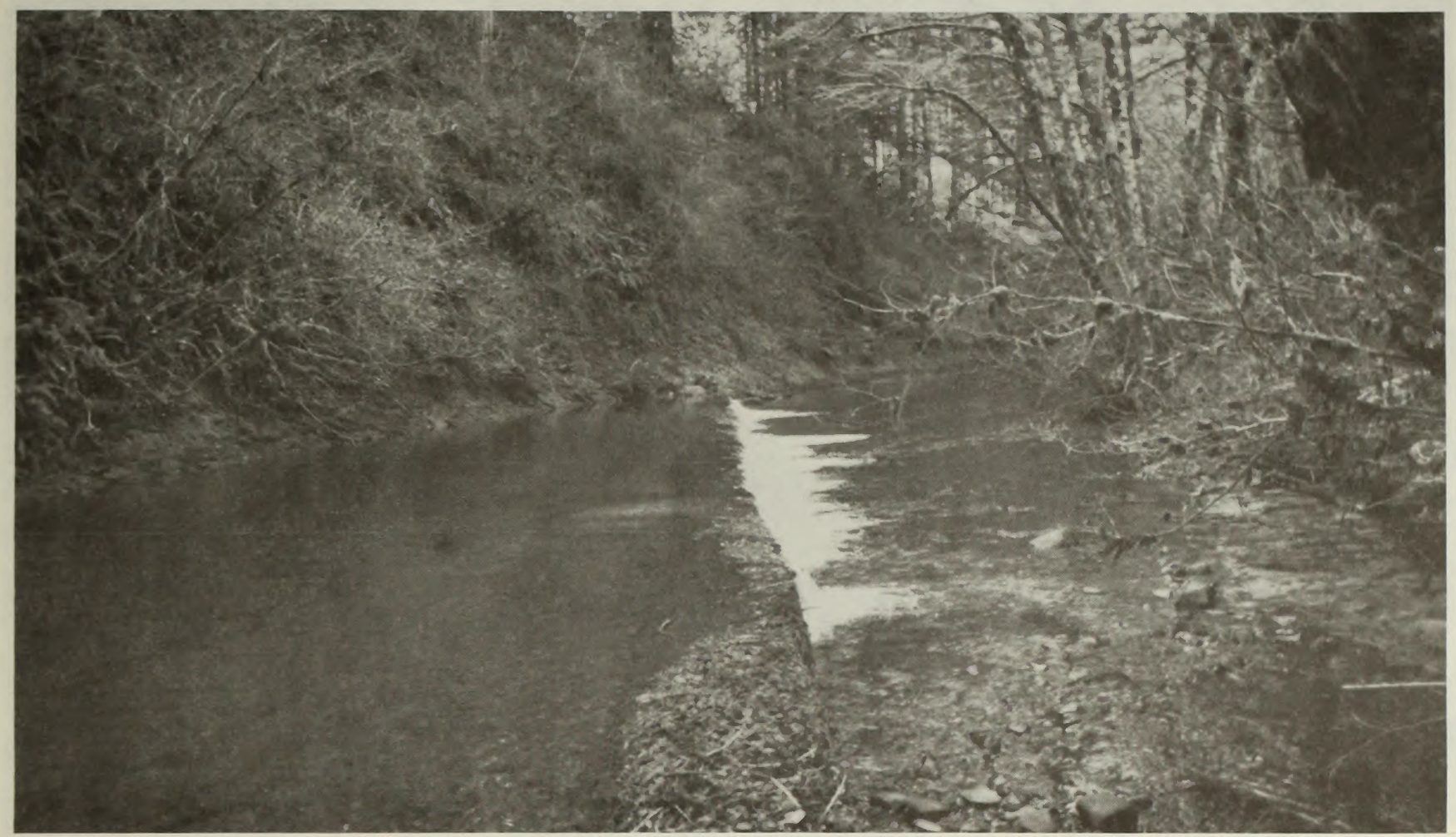

West Fork (Smith River) - Example of a "diagonal" gabion that has worked well on both straight reaches and bends if properly placed.

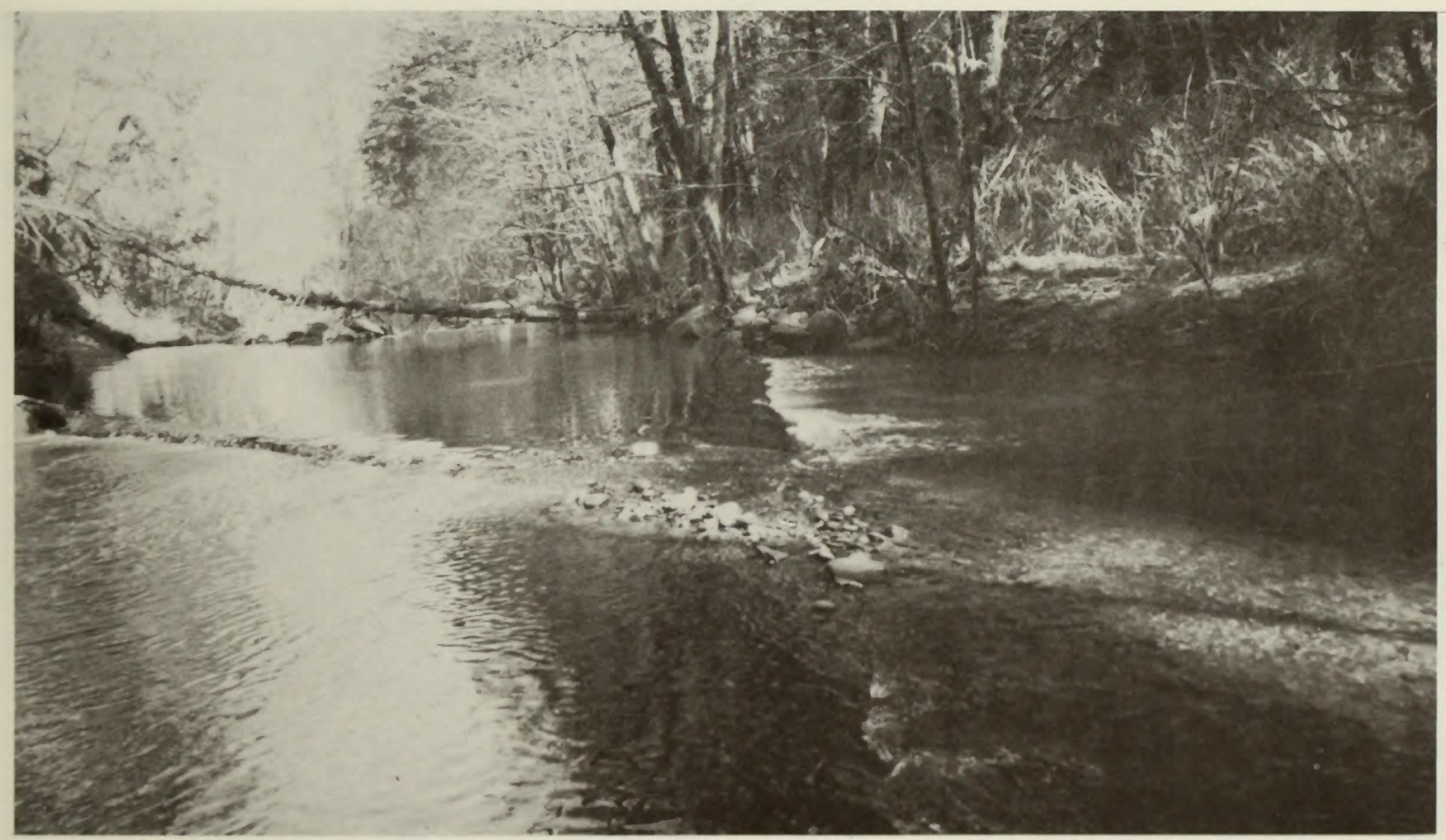

West Fork (Smith River) - Example of a "Y" gabion that has been effective on straight stream reaches. 


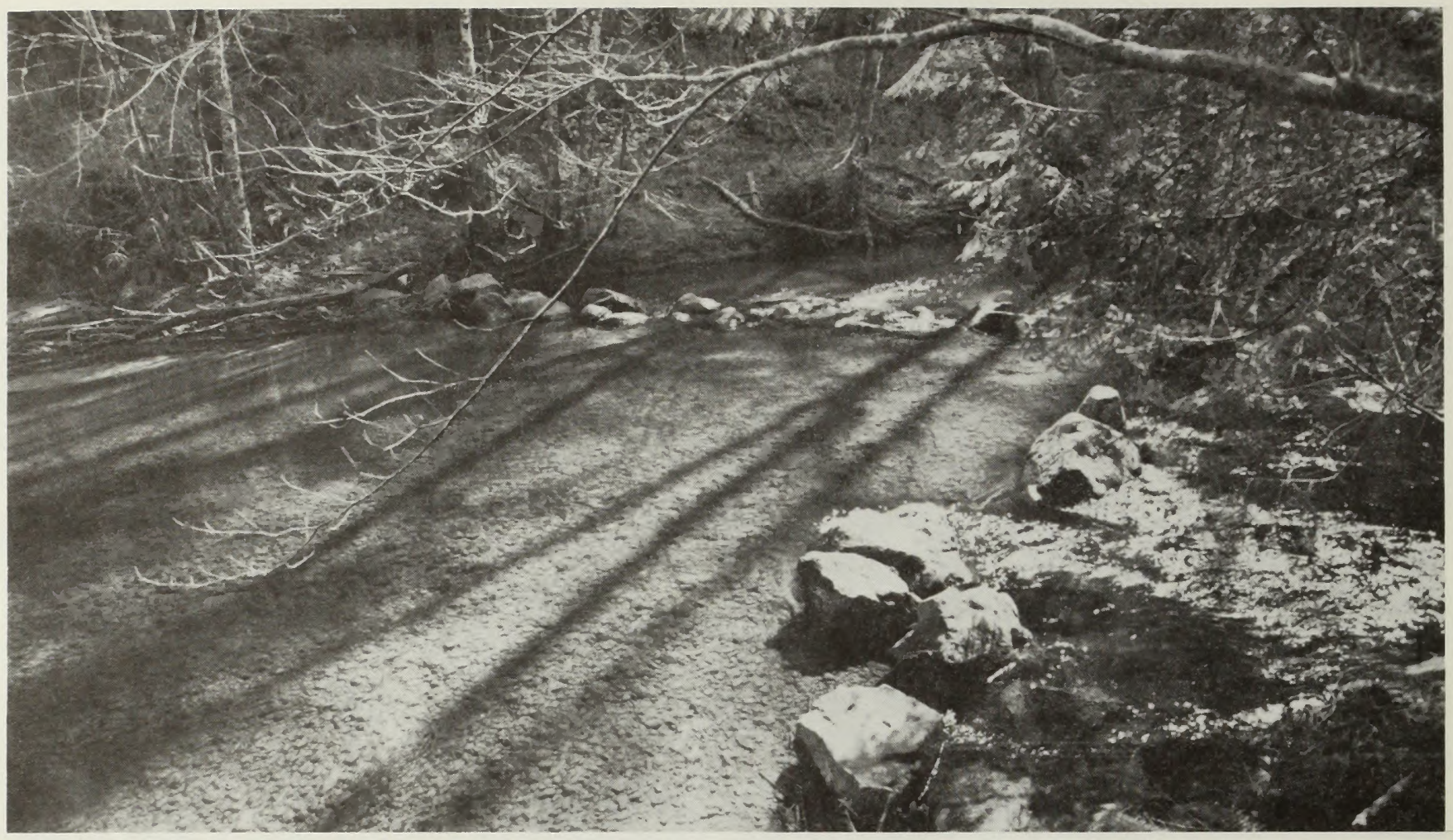

West Fork (Smith River) - A "V" boulder berm designed to create both spawning and rearing habitat. Although the boulders were loosely placed, there has been a heavy accumulation of spawning gravel.

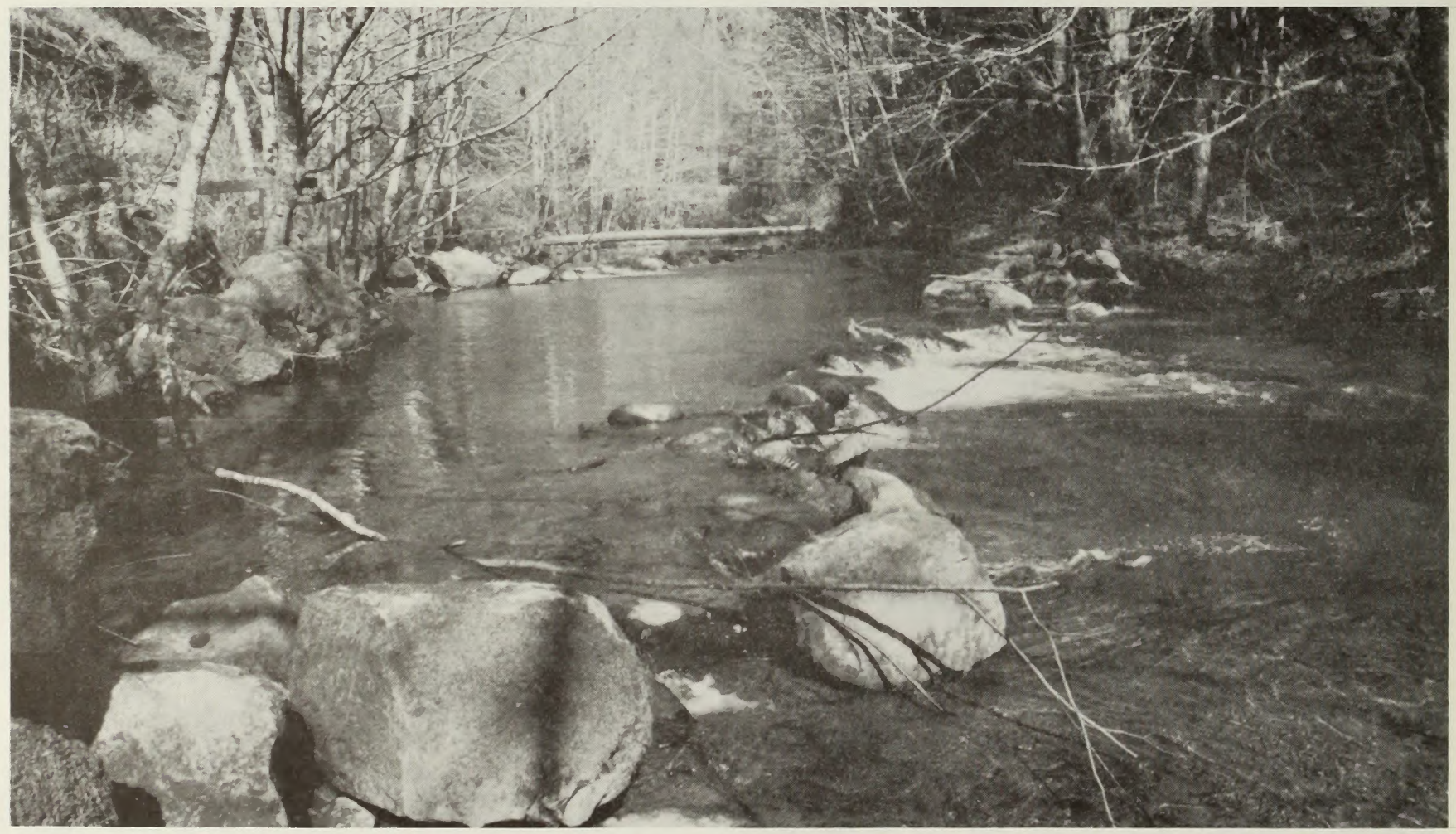

West Fork (Smith River) - An example of a "diagonal" boulder berm. 


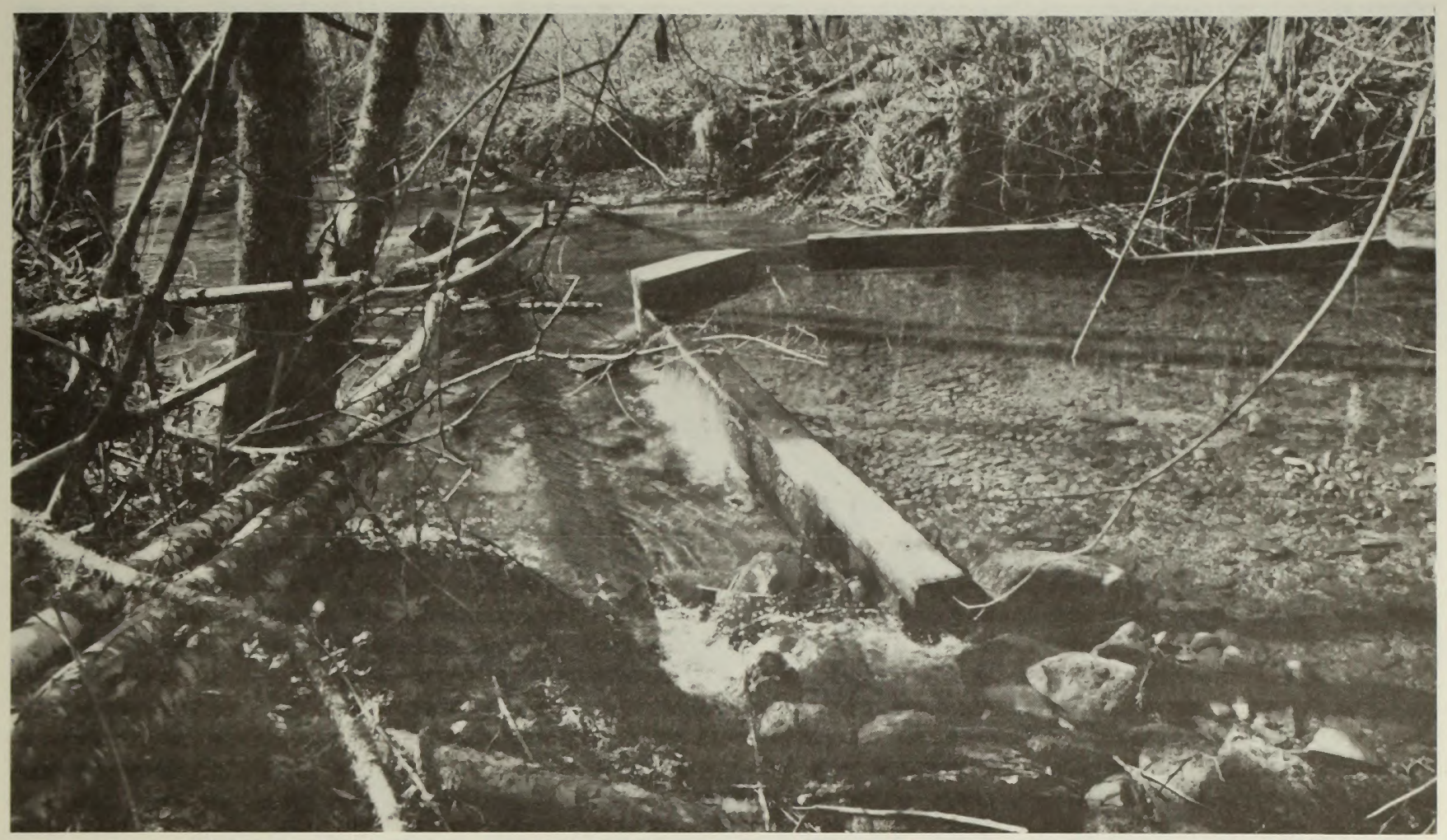

Moore Creek (West Fork, Smith River) - A cedar-board drop structure instalied to create spawning and rearing habitat in a streambed composed primarily of bedrock. Note accretion of spawning gravel and experimental "digger log" device cabled to alders below structure to maintain pool habitat.

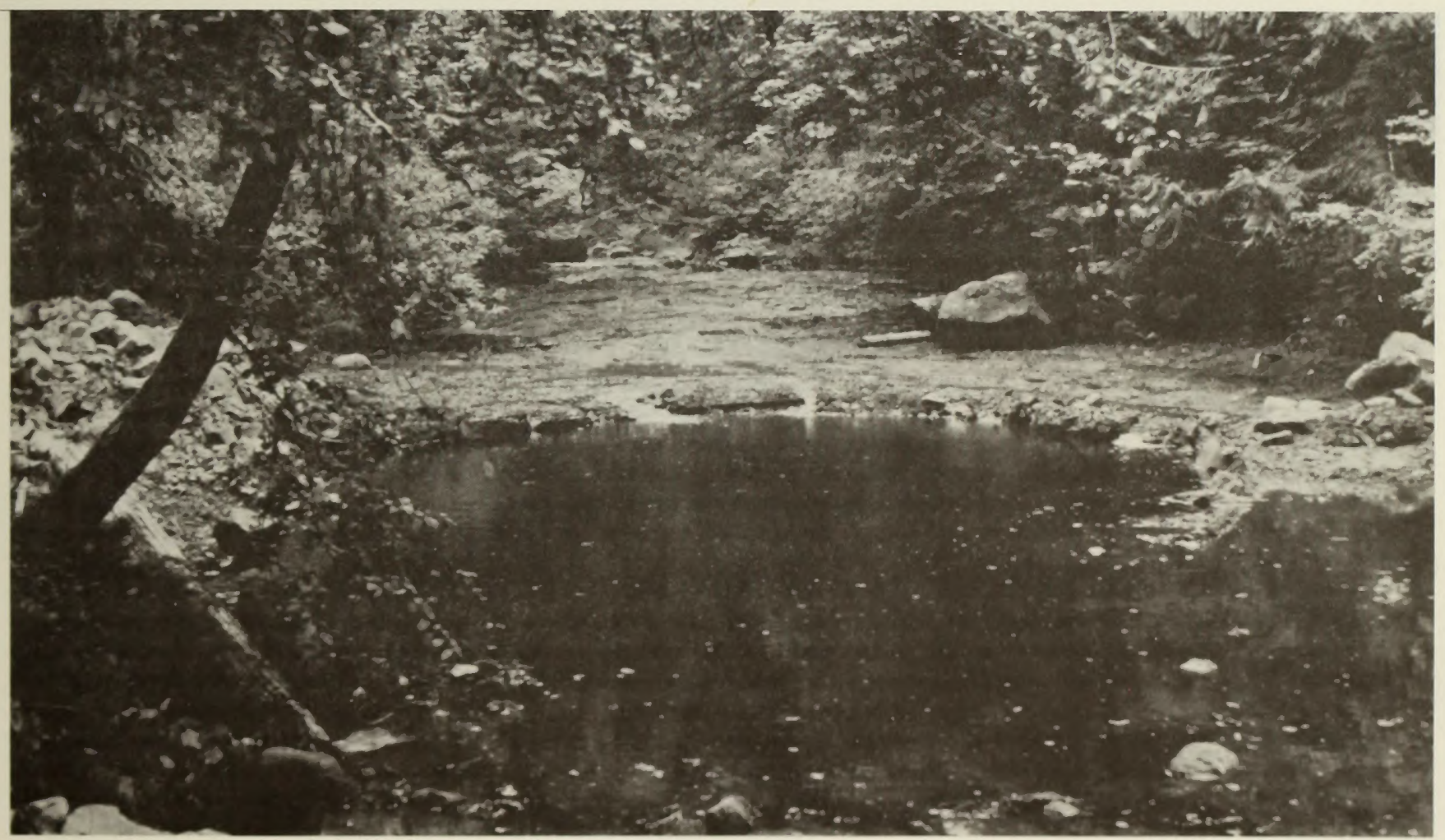

Vincent Creek (Smith River) - a large rearing pool blasted in bedrock streambed to create rearing habitat for anadromous fish. Photo taken at low summer flow. 


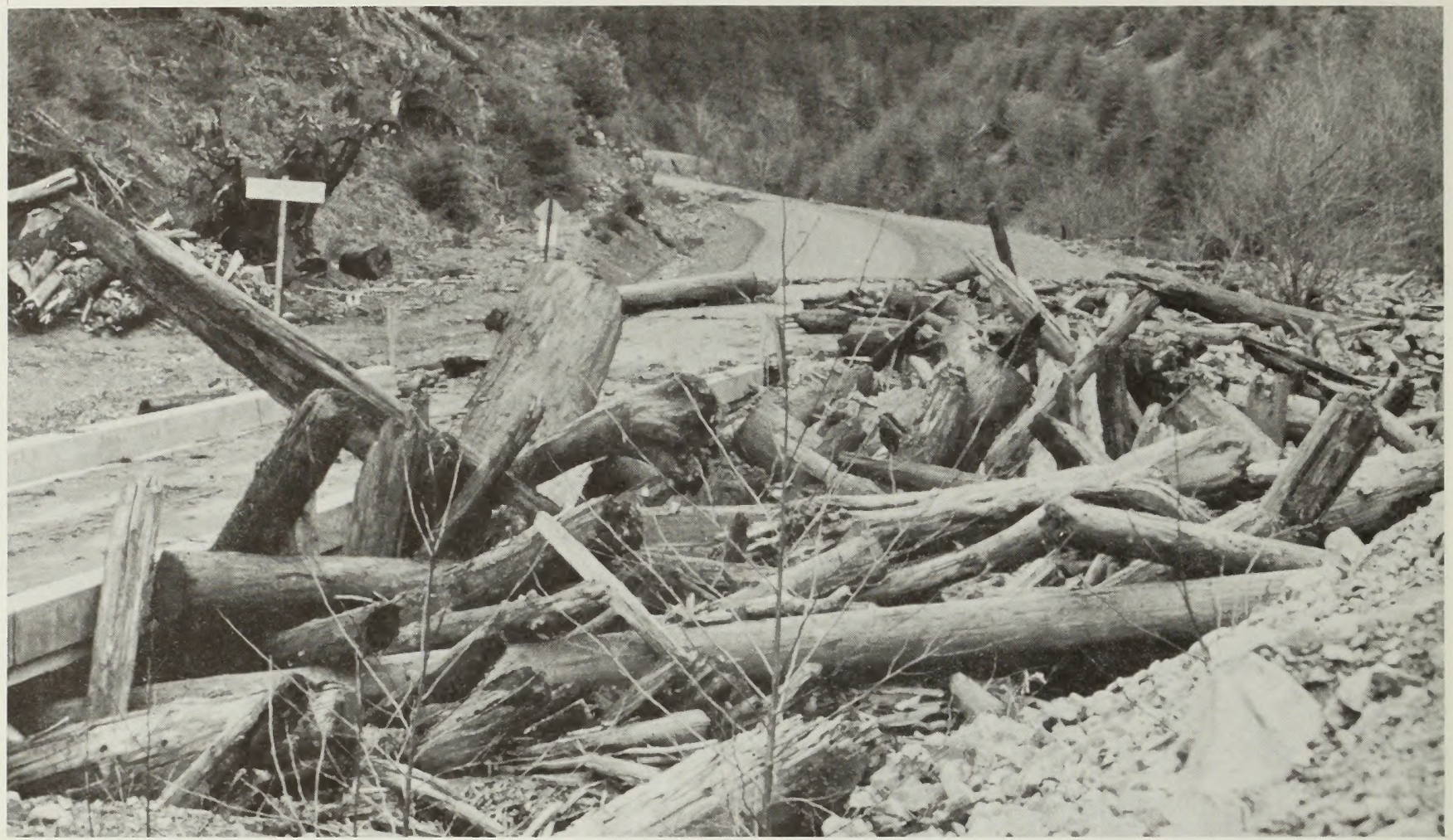

Rock Creek tributary (North Umpqua River) - A log and debris jam caused by winter flooding before removal.

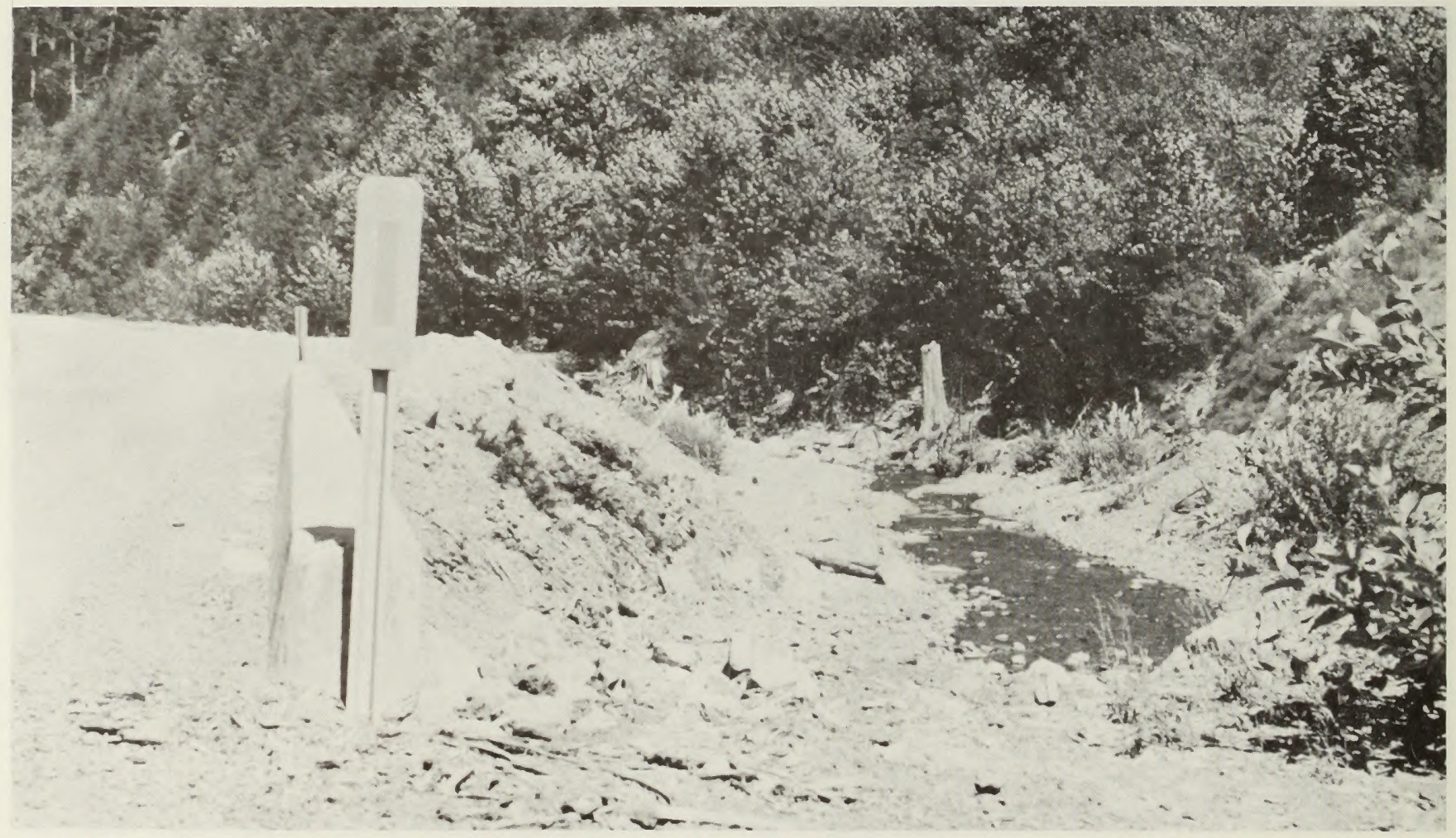

Rock Creek Tributary (after removal) - This type of project work will continue to be done after major floods or debris avalanches when necessary to maintain access for anadromous fish to production areas and/or protect property investments. Some LWM will be left in stream channels during future log jam passage projects to enhance fish production. 


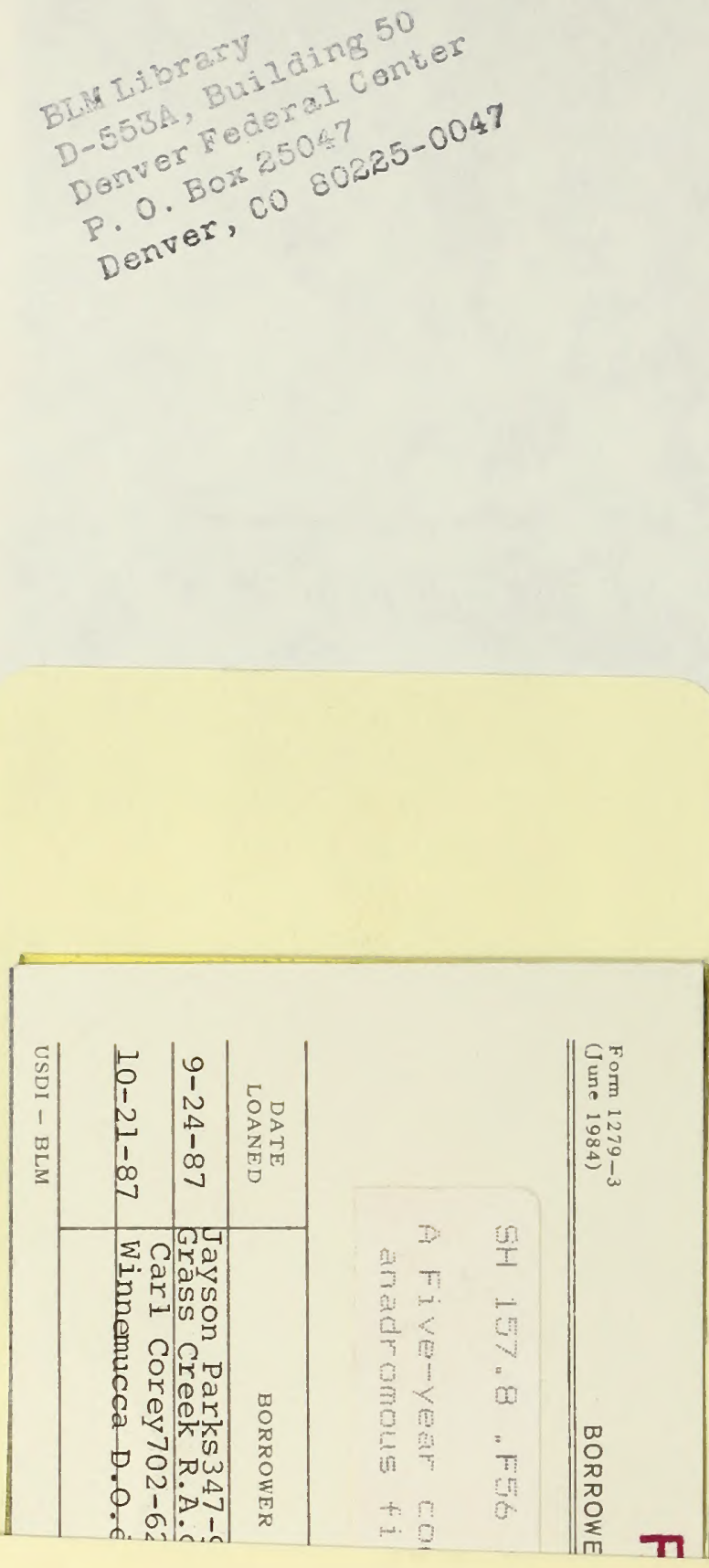

\footnotetext{
BIM Library

D-553A, Building 50

Denver Federal Center

P. O. Box 2504?

Eonver, co 80p?
} 


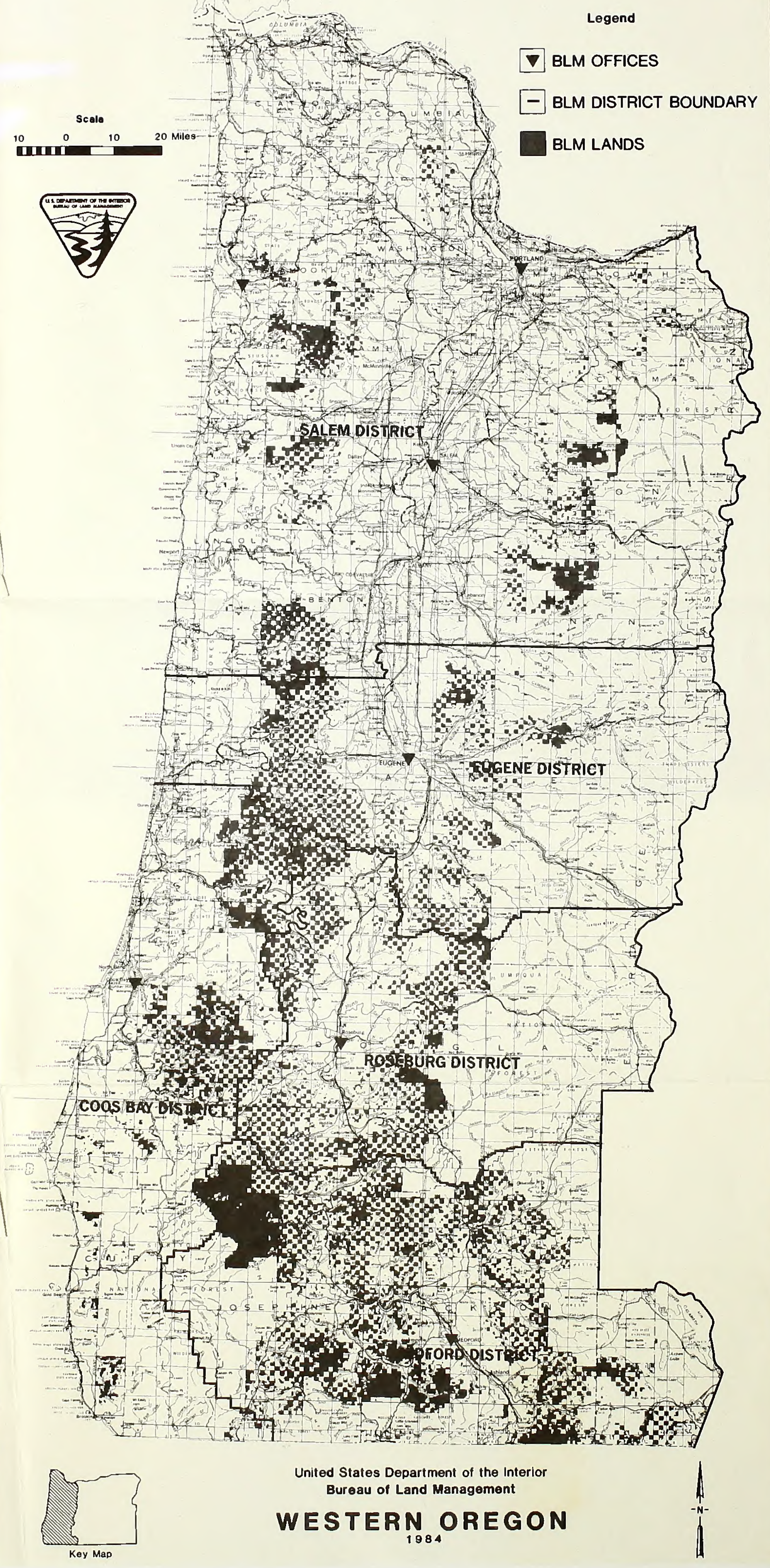


